\title{
THE GOULD'S BELT VERY LARGE ARRAY SURVEY. IV. THE TAURUS-AURIGA COMPLEX
}

\author{
Sergio A. DziB ${ }^{1}$, Laurent Loinard ${ }^{2}$, Luis F. Rodríguez ${ }^{2,3}$, Amy J. Mioduszewski ${ }^{4}$, Gisela N. Ortiz-León ${ }^{2}$, \\ Marina A. Kounkel ${ }^{5}$, Gerardo PeCh ${ }^{2}$, Juana L. Rivera ${ }^{2}$, Rosa M. Torres ${ }^{6}$, Andrew F. Boden ${ }^{7}$, \\ Lee Hartmann $^{5}$, Neal J. Evans II ${ }^{8}$, Cesar Briceño ${ }^{9}$, And John Tobin ${ }^{10}$ \\ ${ }^{1}$ Max Planck Institut für Radioastronomie, Auf dem Hügel 69, D-53121 Bonn, Germany; sdzib@ mpifr-bonn.mpg.de \\ ${ }^{2}$ Centro de Radioastronomía y Astrofísica, Universidad Nacional Autónoma de México Apartado Postal 3-72, 58090 Morelia, Michoacán, Mexico \\ ${ }^{3}$ King Abdulaziz University, P.O. Box 80203, Jeddah 21589, Saudi Arabia \\ ${ }^{4}$ National Radio Astronomy Observatory, Domenici Science Operations Center, 1003 Lopezville Road, Socorro, NM 87801, USA \\ ${ }^{5}$ Department of Astronomy, University of Michigan, 500 Church Street, Ann Arbor, MI 48105, USA \\ ${ }^{6}$ Instituto de Astronomía y Meteorología, Universidad de Guadalajara, Avenida Vallarta No. 2602, Col. Arcos Vallarta, CP 44130 Guadalajara, Jalisco, México \\ ${ }^{7}$ Division of Physics, Math, and Astronomy, California Institute of Technology, 1200 East California Boulevard, Pasadena, CA 91125, USA \\ ${ }^{8}$ Department of Astronomy, The University of Texas at Austin, 1 University Station, C1400, Austin, TX 78712, USA \\ ${ }^{9}$ Cerro Tololo Interamerican Observatory, Casilla 603, La Serena, Chile \\ ${ }^{10}$ Leiden Observatory, Leiden University, P.O. Box 9513, 2300 RA Leiden, The Netherlands \\ Received 2014 November 17; accepted 2014 December 19; published 2015 March 10
}

\begin{abstract}
We present a multi-epoch radio study of the Taurus-Auriga star-forming complex made with the Karl G. Jansky Very Large Array at frequencies of $4.5 \mathrm{GHz}$ and $7.5 \mathrm{GHz}$. We detect a total of 610 sources, 59 of which are related to young stellar objects (YSOs) and 18 to field stars. The properties of $56 \%$ of the young stars are compatible with non-thermal radio emission. We also show that the radio emission of more evolved YSOs tends to be more non-thermal in origin and, in general, that their radio properties are compatible with those found in other starforming regions. By comparing our results with previously reported X-ray observations, we notice that YSOs in Taurus-Auriga follow a Güdel-Benz relation with $\kappa=0.03$, as we previously suggested for other regions of star formation. In general, YSOs in Taurus-Auriga and in all the previous studied regions seem to follow this relation with a dispersion of $\sim 1$ dex. Finally, we propose that most of the remaining sources are related with extragalactic objects but provide a list of 46 unidentified radio sources whose radio properties are compatible with a YSO nature.
\end{abstract}

Key words: radio continuum: stars - radiation mechanisms: non-thermal - radiation mechanisms: thermal techniques: interferometric

Supporting material: machine-readable tables

\section{INTRODUCTION}

Taurus-Auriga is one of the best studied star-forming regions. Indeed, many of the pioneering studies of star formation were based on observation of the young stellar objects (YSOs) and clouds that compose this complex (see Kenyon et al. 2008 for a recent review). The Taurus-Auriga region is particularly appropriate to study individual low-mass YSOs because star formation there occurs in a quiescent environment with low stellar density. This is unlike the situation for stars forming in dense clusters exposed to massive stars such as in the Orion Nebula region. In addition, its relative proximity (140 pc with a depth of up to $30 \mathrm{pc}$; see Loinard et al. 2005, 2007a; Torres et al. 2007, 2009, 2012) makes this complex an ideal candidate for studies of individual YSOs.

Observations in various bands of the electromagnetic spectrum have been obtained for the entire complex (e.g., X-rays by Güdel et al. 2007; optical by Briceño et al. 1993, 1999; infrared by Padgett et al. 2007; near-infrared by Duchêne et al. 2004, and sub-millimeter by Andrews \& Williams 2005). In contrast, however, there are very few radio surveys. This is mainly due to the large solid angle covered by the Taurus-Auriga complex (more than $100 \mathrm{deg}^{2}$; e.g., Figure 1) compared with the typical field of view of radio interferometers $\left(\sim 0.1 \mathrm{deg}^{2}\right)$. In general, radio observations have been devoted to some specific sources of interest. An extensive survey was performed by O'Neal et al. (1990) who pointed 99 fields with the Very Large Array (VLA) at a frequency of $5 \mathrm{GHz}$ to reach a sensitivity of $0.7 \mathrm{mJy}$. These fields contained a total of 119 YSO candidates, but only 9 were detected. Here we present a study with the same approach but with one order of magnitude better sensitivity than those obtained by O'Neal et al. (1990). Additionally, we simultaneously observe at two frequencies and in three different epochs to characterize the spectral index and the variability of the sources.

This research is part of a recently initiated radio survey of YSOs in nearby $(<500 \mathrm{pc})$ star-forming regions (see Dzib et al. 2013a; Kounkel et al. 2014; Ortiz-León et al. 2014, and G. Pech et al. 2015, in preparation for recent results) to characterize their radio emission. Particularly, we are interested in distinguishing between thermal and non-thermal emission of YSOs by measuring the spectral index $\alpha$ (defined such that the flux density depends on frequency as $S_{v} \propto v^{\alpha}$ ) and variability of the flux density. These data will also be used to compare the radio emission properties with the emission at other wavelengths.

\section{OBSERVATIONS}

The observations were obtained with the Karl G. Jansky VLA of the National Radio Astronomy Observatory (NRAO) in its B and BnA configuration. Two frequency sub-bands, each $1 \mathrm{GHz}$ wide, and centered at 4.5 and $7.5 \mathrm{GHz}$, respectively, were recorded simultaneously. The observations were obtained on three different time periods (February 25/26/28 to March 6; April 12/17/20/25, and April 30 to May 1/5/14/22 in 2011) typically separated from one another by a month, see Table 1 for details. This dual frequency, multi-epoch strategy was chosen 


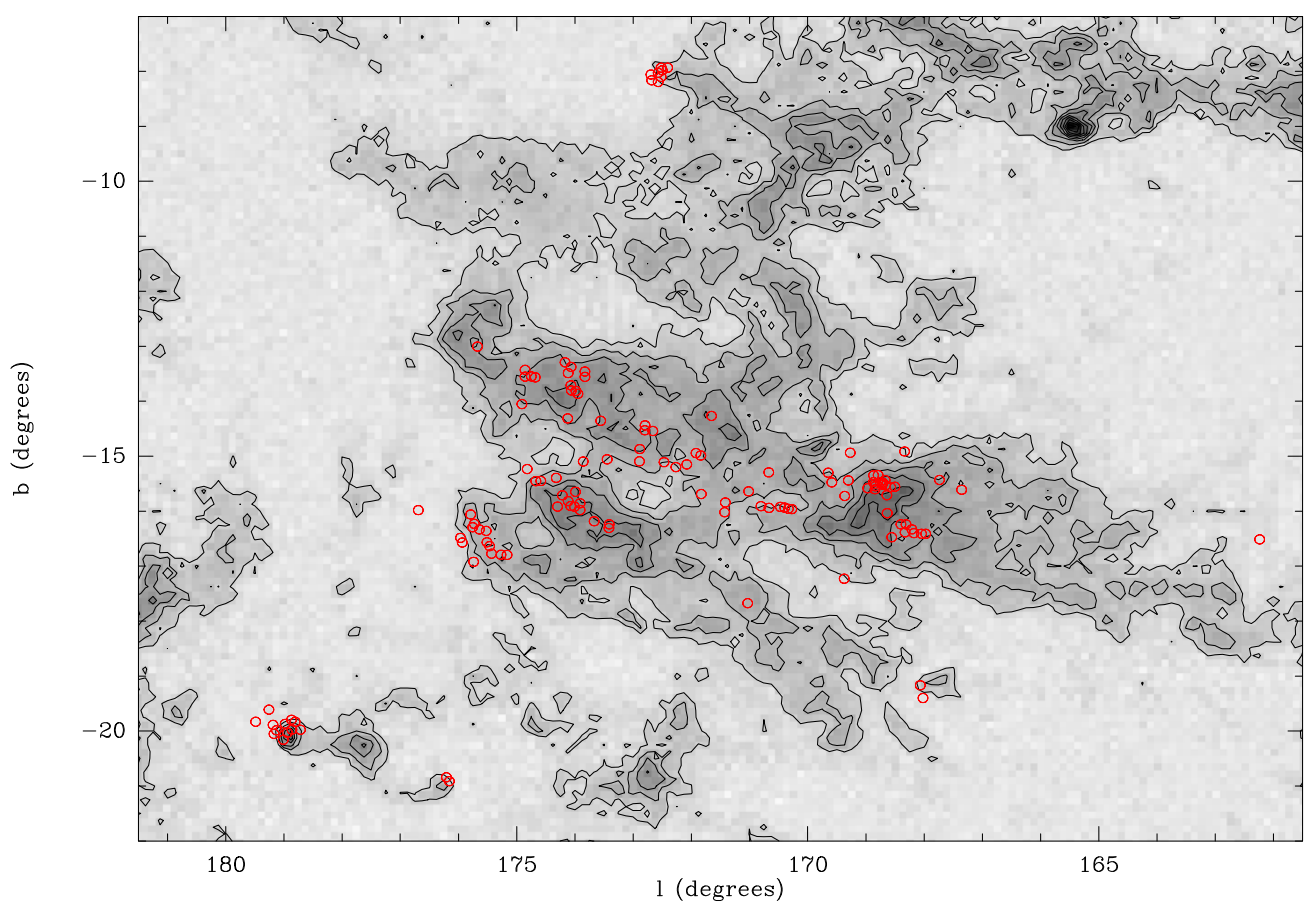

Figure 1. Background: CO extinction map (from Dobashi et al. 2005). Red circles are the VLA observed fields. The diameter of each circle is $10^{\prime}$, i.e., slightly larger than the primary beam used at $7.5 \mathrm{GHz}$, see text.

Table 1

Taurus-Auriga VLA Observations

\begin{tabular}{|c|c|c|c|c|c|c|c|}
\hline \multirow[t]{2}{*}{ Block } & \multirow[t]{2}{*}{ Epoch $^{\mathrm{a}}$} & \multirow{2}{*}{$\begin{array}{c}\text { Date } \\
2011 \\
\\
\text { (dd.mm) }\end{array}$} & \multirow[t]{2}{*}{$\begin{array}{c}\text { VLA } \\
\text { Config. }\end{array}$} & \multicolumn{2}{|c|}{$\begin{array}{c}\text { Synthesized Beam } \\
\left(\theta_{\text {maj }}\left[{ }^{\prime \prime}\right] \times \theta_{\min }\left[{ }^{\prime \prime}\right] ; \text { P.A. }\left[\left[^{\circ}\right]\right)\right.\end{array}$} & \multicolumn{2}{|c|}{$\begin{array}{c}\text { rms Noise } \\
\mu \mathrm{Jy}_{\text {beam }}{ }^{-1}\end{array}$} \\
\hline & & & & $4.5 \mathrm{GHz}$ & $7.5 \mathrm{GHz}$ & $4.5 \mathrm{GHz}$ & $7.5 \mathrm{GHz}$ \\
\hline \multirow[t]{4}{*}{$\bar{A}$} & 1 & 25.02 & $\mathrm{~B}$ & $1.17 \times 1.09 ; 101.7$ & $0.72 \times 0.67 ; \quad 108.4$ & 35 & 29 \\
\hline & 2 & 17.04 & B & $1.15 \times 1.08 ; 104.3$ & $0.69 \times 0.65 ; 112.2$ & 39 & 32 \\
\hline & 3 & 14.05 & $\mathrm{~B} \rightarrow \mathrm{A}$ & $2.69 \times 0.36 ; \quad 58.8$ & $1.35 \times 0.24 ; \quad 59.7$ & 50 & 36 \\
\hline & $C^{\mathrm{b}}$ & & & $1.17 \times 1.10 ;-76.8$ & $0.72 \times 0.67 ;-72.0$ & 30 & 22 \\
\hline \multirow[t]{4}{*}{ B } & 1 & 25.02 & B & $1.62 \times 1.06 ; \quad 84.6$ & $1.02 \times 0.64 ; \quad 84.2$ & 34 & 27 \\
\hline & 2 & 12.04 & $\mathrm{~B}$ & $1.89 \times 1.09 ; \quad 89.3$ & $1.53 \times 0.63 ; \quad 85.2$ & 52 & 40 \\
\hline & 3 & 01.05 & $\mathrm{~B}$ & $1.30 \times 0.97 ;-20.6$ & $0.69 \times 0.65 ;-49.7$ & 42 & 30 \\
\hline & $C$ & & & $1.37 \times 1.11 ; \quad 90.2$ & $0.86 \times 0.65 ; \quad 86.7$ & 26 & 19 \\
\hline \multirow[t]{4}{*}{$\mathrm{C}$} & 1 & 26.02 & B & $1.10 \times 1.02 ;-21.9$ & $0.68 \times 0.63 ;-19.0$ & 35 & 27 \\
\hline & 2 & 20.04 & B & $1.66 \times 1.02 ; \quad 84.5$ & $1.03 \times 0.63 ; \quad 85.6$ & 38 & 30 \\
\hline & 3 & 05.05 & $\mathrm{~B}$ & $1.66 \times 1.10 ; \quad 81.5$ & $1.02 \times 0.65 ; \quad 86.0$ & 39 & 30 \\
\hline & $C$ & & & $1.30 \times 1.07 ; \quad 89.3$ & $0.80 \times 0.66 ; \quad 90.4$ & 22 & 18 \\
\hline \multirow[t]{4}{*}{$\mathrm{D}$} & 1 & 28.02 & B & $1.12 \times 1.05 ; \quad 44.7$ & $0.65 \times 0.62 ; \quad 42.1$ & 34 & 30 \\
\hline & 2 & 25.04 & B & $1.11 \times 1.01 ;-24.2$ & $0.68 \times 0.63 ;-22.7$ & 35 & 25 \\
\hline & 3 & 30.04 & B & $1.50 \times 1.14 ; \quad 69.6$ & $0.89 \times 0.70 ; \quad 74.8$ & 45 & 36 \\
\hline & $C$ & & & $1.13 \times 1.09 ; \quad 56.5$ & $0.68 \times 0.66 ; \quad 60.5$ & 21 & 17 \\
\hline \multirow[t]{4}{*}{$\mathrm{E}$} & 1 & 06.03 & $\mathrm{~B}$ & $1.38 \times 1.07 ;-84.2$ & $0.85 \times 0.65 ;-84.3$ & 32 & 26 \\
\hline & 2 & 25.04 & $\mathrm{~B}$ & $1.11 \times 1.02 ;-42.0$ & $0.66 \times 0.60 ;-35.7$ & 39 & 32 \\
\hline & 3 & 22.05 & $\mathrm{~B} \rightarrow \mathrm{A}$ & $1.68 \times 0.36 ;-69.9$ & $1.06 \times 0.22 ;-69.8$ & 40 & 31 \\
\hline & $C$ & & & $1.32 \times 0.53 ;-71.2$ & $0.82 \times 0.33 ;-70.0$ & 24 & 19 \\
\hline
\end{tabular}

Notes.

${ }^{\text {a }} C$ indicates parameters measured in the images after combining the epochs.

${ }^{\mathrm{b}}$ Only epoch 1 and 2 were concatenated due to systematically lower flux densities values on epoch 3 . See also text.

to enable the characterization of the spectral index and variability of the detected sources, and to help in the identification of the emission mechanisms (thermal versus non-thermal).

For our study, we observed 127 different target fields distributed across the cloud complex (Figure 1). The fields were chosen to cover previously known YSOs. In 33 of those fields, we could observe more than one YSO target, while in the remaining 94 fields, only one YSO was targeted. In most cases, the infrared evolutionary class (i.e., Classes I, II, or III) or T Tauri evolutionary status (classical or weak line) of the targeted sources was known from the literature. Due to the number of observed fields we divided them in five different blocks, named from A to E, and each observation session ran for two hours. We obtained a total of 15 observation sessions for the Taurus-Aurigae region, and they are summarized in Table 1. 
Table 2

Radio Sources Detected in Taurus-Auriga

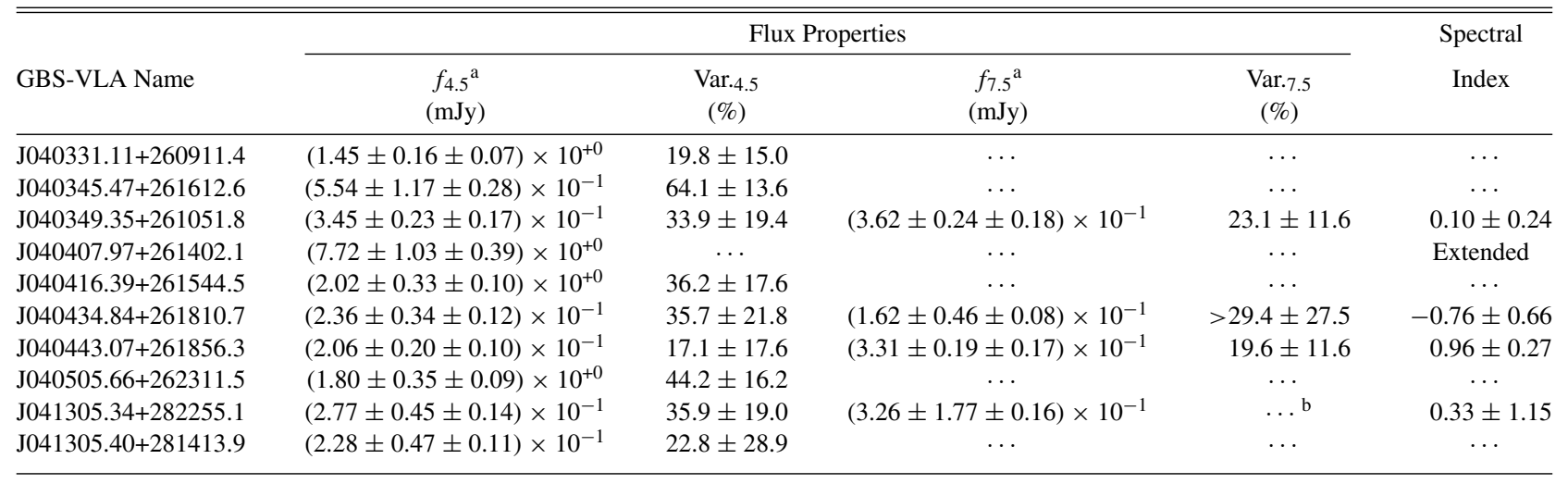

Notes.

a The two given errors correspond to (1) statistical noise in the images and (2) systematic uncertainty from possible errors in flux calibration.

b Source not detected at three times the noise level on individual epochs, but detected on the image of the concatenated epochs.

(This table is available in its entirety in machine-readable form.)

Because of the large total area covered by the observations, three different phase calibrators were used: J0449+1121, $\mathrm{J} 0403+2600$ and $\mathrm{J} 0443+3441$, depending on the position of the target fields. The flux calibrator, for all the observations, was the quasar 3C 147 that was also used as the bandpass calibrator.

Each observing session was organized as follows. The standard flux calibrator 3C 147 was first observed for $\sim 10$ minutes. We subsequently spent one minute on the phase calibrator followed by a series of three target pointings, spending three minutes on each, closing the cycle with a new observation of one minute on the phase calibrator. This phase calibrator/target sequence was repeated until all target fields were observed. Thus, three minutes were spent on each target field for each epoch. The data were edited, calibrated, and imaged in a standard fashion using the Common Astronomy Software Applications package (CASA). The data calibration was the same as that performed for the Ophiuchus observations reported in Dzib et al. (2013a) and is not repeated here. When imaging, the minimum level of the primary beam used was at a response of $20 \%$. The final images covered circular areas of 8.8 and 14.3 arcmin in diameter, for the 7.5 and $4.5 \mathrm{GHz}$ sub-bands, respectively, and were corrected for the effects of the position-dependent primary beam response. The noise levels reached for each individual observation was about $\sim 40 \mu \mathrm{Jy}$ and $\sim 30 \mu \mathrm{Jy}$, at $4.5 \mathrm{GHz}$ and $7.5 \mathrm{GHz}$, respectively (see Table 1 for the individual values at each epoch).

In two epochs we detected problems. First, for the third epoch of Block A we observed systematically lower flux densities for the detected sources, compared to the first two epochs, thus we discard this epoch for our main analysis. Still, we used this epoch to search any extra detections. Second, at the beginning of the observation of the second epoch of Block C, some scans were missed. Despite these problems, our analysis is not affected.

The visibilities of the three, or two, observations obtained for each field were concatenated to produce a new image with a lower noise level (of about $\sim 25 \mu \mathrm{Jy}$ at $4.5 \mathrm{GHz}$ and $\sim 18 \mu \mathrm{Jy}$ at $7.5 \mathrm{GHz}$ ). The angular resolution of $\sim 1^{\prime \prime}$ (see the synthesized beam sizes in Table 1) allows an uncertainty in position of $\sim 0^{\prime \prime} .1$ or better. To test for circular polarization we produced images of the $V$ Stokes parameter in the inner quarter (in area) of the primary beam at each frequency. At larger distances from the field center, polarization measure- ments become unreliable as beam squint (the separation of the $R$ and $L$ beams on the sky) can create artificial circular polarization signals.

\section{RESULTS}

In the observed area, there is a total of 196 known YSOs. The first step was the identification of radio sources in the observed fields. We follow the procedure and criteria presented by Dzib et al. (2013a) who consider a detection as firm if the sources have a flux larger than $4 \times \sigma_{\text {noise }}$ level and there is a counterpart know at other wavelength, else we require a $5 \times \sigma_{\text {noise }}$ level. The identification was done using the images corresponding to the concatenation of the observed epochs, which provides the highest sensitivity. From this, a total of 609 sources were detected (see Table 2). From these sources 215 were only detected in the $4.5 \mathrm{GHz}$ sub-band, while six were only detected in the $7.5 \mathrm{GHz}$ sub-band. The remaining 388 sources were detected in both sub-bands. We also used the third epoch of Block A to search for any additional detection. One source, related to the YSO Coku HP Tau G3, was detected at both subbands in this epoch, but not detected in the concatenated map of the two first epochs of Block A. Consequently, the total number of detected sources in this survey is 610 . To reflect the fact that these sources were found as part of the Gould's Belt VLA Survey, a source with coordinates hhmmss.ss+ddmmss.s will be named GBS-VLA Jhhmmss.ss+ddmmss.s.

The fluxes of each source at 4.5 and $7.5 \mathrm{GHz}$ are given in Columns 2 and 4 of Table 2. Two main sources of uncertainties on the fluxes are included and given independently in Table 2: (1) the error that results from the statistical noise in the images and (2) a systematic uncertainty of 5\% resulting from possible errors in the absolute flux calibration (e.g., Perley \& Buttler 2013). We additionally take into account errors produced by pointing errors of the primary beam (following Dzib et al. 2014), whose effects are more noticeable for sources detected at the edges of the primary beam. These errors were added in quadrature to the statistical errors. An estimation of the radio spectral index of each source (given in Column 6 of Table 2) was obtained from the fluxes measured in each sub-band (at 4.5 and $7.5 \mathrm{GHz}$ ). To calculate the errors on the spectral indices, also given in Column 6 of Table 2, the sources of errors on the 
Table 3

Sources Detected in Circular Polarization

\begin{tabular}{llcc}
\hline \hline GBS-VLA Name & Source Type & $\begin{array}{c}4.5 \mathrm{GHz} \text { Polz } \\
(\%)\end{array}$ & $\begin{array}{c}7.5 \mathrm{GHz} \text { Polz } \\
(\%)\end{array}$ \\
\hline J041327.23+281624.4 & YSO/Class II & $36.3(\mathrm{~L})$ & $<30.5$ \\
J041628.11+280735.4 & YSO/Unknown & $9.6(\mathrm{~L})$ & $18.8(\mathrm{~L})$ \\
J041831.12+282715.9 & YSO/Class III & $<6.5$ & $8.7(\mathrm{R})$ \\
J041840.62+281915.3 & YSO/Class II & $32.0(\mathrm{~L})$ & $<29.9$ \\
J041847.04+282007.2 & YSO/Class III & $3.1(\mathrm{~L})$ & $5.6(\mathrm{~L})$ \\
J041941.28+274947.9 & YSO/Unknown & $5.1(\mathrm{~L})$ & $<2.6$ \\
J042159.43+193205.7 & YSO/Class II & $69.7(\mathrm{~L})$ & $\ldots$ \\
J042203.15+282538.8 & YSO/Class III & $21.3(\mathrm{~L})$ & $30.8(\mathrm{~L})$ \\
J043140.09+181356.7 & YSO/Class III & $35.8(\mathrm{~L})$ & $31.2(\mathrm{~L})$ \\
J043214.58+182014.6 & YSO/Class III & $17.8(\mathrm{R})$ & $<6.6$ \\
J043542.05+225222.4 & Star & $28.8(\mathrm{R})$ & $\ldots$ \\
\hline & & & $\ldots$
\end{tabular}

flux at each frequency were added in quadrature and the final error was obtained using standard error propagation theory.

Once the sources were identified in the concatenated images, we searched for them in the images obtained from the individual epochs. An estimate of the level of variability of the sources was obtained by comparing the fluxes measured at the three epochs. Specifically, we calculated, for each source and at each frequency, the difference between the highest and lowest measured fluxes, and normalized by the maximum flux. The resulting values, expressed as percentages are given in Columns 3 and 5 of Table 2. To calculate the errors on the variability, also given in Columns 3 and 5 of Table 2, the sources of errors on the flux at each epoch were added in quadrature and the final error was obtained using standard error propagation theory. We will define as a highly variable sources those that change their flux density by at least a factor of two between the maximum and minimum measured fluxes (i.e., a variability larger than $50 \%$ ) and that the result is significant above a three sigma level. Circular polarization was confidently detected in 11 radio sources (Table 3 ).

Having identified the radio sources in the region mapped, and having derived their variability, polarization, and spectral index, our next step was to try to determine which type of object they are associated with. In our specific case, the two overwhelmingly dominant possibilities are young stars and extragalactic sources. ${ }^{11}$ We searched the literature for previous radio detections, and for counterparts at X-ray, optical, near-infrared, and mid-infrared wavelengths. The search was done in SIMBAD, and accessed all the major catalogs (listed explicitly in the footnote of Table 4). We considered a radio source associated with a counterpart at another wavelength if the separation between the two was below the combined uncertainties of the two data sets. This was about 1.0 arcsec for the optical and infrared catalogs, but could be significantly larger for some of the radio catalogs (for instance, the NVSS has a positional uncertainty of about 5 arcsec).

We found that only 120 of the sources detected here had previously been reported at radio wavelengths (Column 7 of Table 4), while the other 491 are new radio detections. On the other hand, we found a total of 270 counterparts at other wavelengths. In the literature, 18 are classified as field stars, 49 as extragalactic, 1 is classified as both star or extragalactic in different surveys, 49 are classified as YSOs, 11 are classified as both YSO and extragalactic, and the remaining 143 are unclassified. Note that 56 sources were previously known at radio wavelengths and do not have known counterparts at other frequencies. As a consequence, the number of sources that were previously known (at any frequency) is 327, while 284 of the sources in our sample are reported here for the first time.

\section{DISCUSSION}

\subsection{Background Sources}

A large number of the sources detected here must correspond to background quasars seen in the direction of our targets. We estimate that number as follows. First, we take into account the fact that at the edges of the fields, the sensitivity is lower than at their centers. Following the Equation (A11) from Anglada et al. (1998), who discuss this effect, we obtain that the rough number of expected background sources with fluxes above $S_{0, v}$ in each

\footnotetext{
${ }^{11}$ However, we cannot fully rule out the possibility that other objects might contaminate the sample (a compact planetary nebula for instance).
}

Table 4

Radio Sources with Known Counterparts

\begin{tabular}{|c|c|c|c|c|c|c|c|}
\hline \multirow[t]{2}{*}{ GBS-VLA Name } & \multirow{2}{*}{$\begin{array}{l}\text { Other } \\
\text { Names }\end{array}$} & \multirow[t]{2}{*}{ X-Ray ${ }^{\mathrm{a}}$} & \multicolumn{3}{|c|}{ Infrared $^{\mathrm{b}}$} & \multirow[t]{2}{*}{ Radio $^{c}$} & \multirow{2}{*}{$\begin{array}{c}\text { Object } \\
\text { Type }\end{array}$} \\
\hline & & & SST & $2 \mathrm{M}$ & WISE & & \\
\hline J040349.35+261051.8 & 2MASS J04034930+2610520 & XEST & $\mathrm{Y}$ & $\mathrm{Y}$ & $\mathrm{Y}$ & $\ldots$ & YSO \\
\hline J040416.39+261544.5 & WISE J040416.33+261545.2 & $\ldots$ & $\ldots$ & $\ldots$ & $\mathrm{Y}$ & $\ldots$ & $\ldots$ \\
\hline J040434.84+261810.7 & WISE J040434.83+261810.5 & & $\ldots$ & $\ldots$ & $\mathrm{Y}$ & $\ldots$ & $\ldots$ \\
\hline J040443.07+261856.3 & IRAS $04016+2610$ & XEST & $\ldots$ & $\mathrm{Y}$ & $\mathrm{Y}$ & $\ldots$ & YSO \\
\hline J040505.66+262311.5 & WISE J040505.68+262311.2 & $\ldots$ & $\ldots$ & $\ldots$ & $\mathrm{Y}$ & NVSS & $\ldots$ \\
\hline J041305.34+282255.1 & WISE J041305.31+282255.1 & $\ldots$ & $\mathrm{Y}$ & $\mathrm{Y}$ & $\mathrm{Y}$ & $\ldots$ & $\ldots$ \\
\hline J041305.40+281413.9 & $\ldots$ & $\ldots$ & $\ldots$ & $\ldots$ & $\ldots$ & NVSS & $\ldots$ \\
\hline $\mathrm{J} 041305.45+281414.7$ & $\ldots$ & $\ldots$ & $\ldots$ & $\ldots$ & $\ldots$ & NVSS & $\ldots$ \\
\hline J041314.16+281910.3 & V1095 Tau & XEST & $\mathrm{Y}$ & $\mathrm{Y}$ & $\mathrm{Y}$ & $\ldots$ & YSO \\
\hline J041327.23+281624.4 & V1096 Tau & XEST & $\mathrm{Y}$ & $\mathrm{Y}$ & $\mathrm{Y}$ & $\ldots$ & YSO \\
\hline
\end{tabular}

Notes.

${ }^{\mathrm{a}}$ XEST = Güdel et al. (2007); 1RXS = Voges et al. (1999); RX = Neuhaeuser et al. (1995) and BFR2003 = Bally et al. (2003).

b SST = Padgett et al. (2007); $2 \mathrm{M}=$ Cutri et al. (2003) and WISE = Cutri et al. (2013).

${ }^{\mathrm{c}}$ NVSS = Condon et al. (1998); BW = Becker \& White (1985); SBS = Skinner et al. (1993); OFMM = O'Neal et al. (1990); LRDRG = Loinard et al. (2007b); RAR = Rodríguez et al. (1995); CB = Cohen \& Bieging (1986); ARC92 = Anglada et al. (1992); RRAB = Reipurth et al. (2004); B96 = Bontemps (1996); GRL2000 = Giovanardi et al. (2000) and RR98 = Rodríguez \& Reipurth (1998).

(This table is available in its entirety in machine-readable form.) 
field and for each sub-band are:

$$
\begin{aligned}
\langle N\rangle_{4.5 \mathrm{GHz}} & =1.21\left(\frac{S_{0,4.5 \mathrm{GHz}}}{\mathrm{mJy}}\right)^{-0.75} \text { and } \\
\langle N\rangle_{7.5 \mathrm{GHz}} & =0.35\left(\frac{S_{0,7.5 \mathrm{GHz}}}{\mathrm{mJy}}\right)^{-0.75} .
\end{aligned}
$$

Using these formulations and $S_{0, v}=5 \times \sigma_{\text {noisev }}$, we expect in our 127 fields a total of $731 \pm 27$ background sources at the $4.5 \mathrm{GHz}$ sub-band and $271 \pm 17$ at the $7.5 \mathrm{GHz}$ sub-band. The range of expected values was estimated assuming a Poisson distribution. Since in our survey we have considerable overlap of the fields, in particular at $4.5 \mathrm{GHz}$, the expected number of sources at $4.5 \mathrm{GHz}$ can be considered as an upper limit. From our detected sources not related to YSO or stars we counted 530 and 316 in the $4.5 \mathrm{GHz}$ and $7.5 \mathrm{GHz}$ sub-bands, respectively. This strongly suggests that most of the unidentified sources reported here are background sources.

To analyze this possibility further, we note that extragalactic radio sources are expected to show little variability (typically $\sim 10 \%$ on a timescale of $1 \mathrm{yr}$ ) and to have negative spectral indices. We have used these characteristics to try and separate background sources from YSO candidates in previous studies (e.g., Dzib et al. 2013a; Kounkel et al. 2014 and Ortiz-León et al. 2014). However, there are well known exceptions of extragalactic radio sources that do not follow the typical behavior (e.g., Heeschen 1984 \& O'Dea 1998) and that we now consider in more detail.

Lovell et al. (2008) found that around half of the flat spectrum extragalactic sources at centimeter wavelengths show significant variability on the scale of days to hours. These variations are attributed mainly to interstellar scintillation by material in our own Galaxy (e.g., Heeschen \& Rickett 1987; Lovell et al. 2008 and Koay et al. 2011). We looked through the data of Lovell et al. (2008) and found that less than $0.6 \%$ of their sources exhibit high variability, using our variability criteria, between two of their four epochs separated from one another by four months. Assuming that our sources follow the same trend would indicate that only three of our background sources should be highly variable. Thus, high variability is a good indicator to separate the YSO population from the background sources.

On the other hand, Gigahertz Peaked-Spectrum (GPS) and High Frequency Peakers (HFP) are extragalactic sources that have convex radio spectra peaking at frequencies up to $5 \mathrm{GHz}$ for GPS sources (see O'Dea 1998 for a review) and above $22 \mathrm{GHz}$ for HFP (Dallacasa et al. 2000). Thus, the spectral index for these sources in the observed frequencies can be flat or positive. About $10 \%$ of the entire population of radio-bright background sources are GPS, while $20 \%$ of these have their peak above $4 \mathrm{GHz}$ (see O'Dea 1998). Finally, the analysis by Dallacasa et al. (2000) shows that roughly $3 \%$ of their initial sources are HFP. Using these results we expect that around 21 of the background sources will have flat or positive spectral indices. Thus, spectral index is also a useful quantity to separate YSOs from background sources, but it is somewhat less reliable than variability.

In our sample of 530 possible background sources, 50 are either highly variable $\left(3 \times \sigma_{\text {Var. }}\right.$ level $)$ or have a flat or positive spectral index value (whitin the range given by $\alpha \pm \sigma_{\alpha}$ ). Four of these were previously known to be extragalactic sources. The remaining 46 sources are listed in Table 5. From the previous arguments, we expect about 24 extragalactic sources with these characteristics. Thus, about half of the sources in Table 4 are
Table 5

\begin{tabular}{|c|c|c|c|}
\hline GBS-VLA Name & $\begin{array}{c}\text { Variability }_{4.5 \mathrm{GHz}} \\
(\%)\end{array}$ & $\begin{array}{c}\text { Variability } 7.5 \mathrm{GHz} \\
(\%)\end{array}$ & Spectral Index \\
\hline $\mathrm{J} 040345.47+261612.6$ & $64.1 \pm 13.6$ & & \\
\hline J041305.45+281414.7 & $70.2 \pm 13.8$ & $\cdots$ & $\ldots$ \\
\hline J041334.03+292255.3 & $>60.7 \pm 16.7$ & $\cdots$ & $\cdots$ \\
\hline J041412.58+281155.7 & $38.9 \pm 18.0$ & $40.6 \pm 21.0$ & $0.42 \pm 0.50$ \\
\hline J041437.95+264604.3 & $13.2 \pm 12.7$ & $39.3 \pm 14.6$ & $1.12 \pm 0.35$ \\
\hline $\mathrm{J} 041454.56+264716.2$ & $>59.9 \pm 15.2$ & $16.1 \pm 38.1$ & $-0.51 \pm 0.55$ \\
\hline J041515.93+291244.5 & $>68.2 \pm 13.8$ & $\ldots$ & $\ldots$ \\
\hline J041821.80+282956.3 & $>44.3 \pm 23.9$ & $>15.0 \pm 28.3$ & $1.06 \pm 0.90$ \\
\hline J041825.42+252156.4 & $47.0 \pm 14.2$ & $>76.8 \pm 17.5$ & $-1.53 \pm 1.08$ \\
\hline $\mathrm{J} 041840.27+282036.0$ & $>61.1 \pm 13.9$ & $10.7 \pm 37.2$ & $-0.63 \pm 0.68$ \\
\hline J041844.85+282804.4 & $52.2 \pm 16.8$ & $>45.9 \pm 29.0$ & $0.17 \pm 0.86$ \\
\hline J041935.50+290628.4 & $34.3 \pm 21.7$ & $\ldots$ a & $<0.01 \pm 0.14$ \\
\hline $\mathrm{J} 042117.65+270151.0$ & $31.9 \pm 9.1$ & $52.2 \pm 16.1$ & $-1.23 \pm 0.38$ \\
\hline $\mathrm{J} 042124.18+270025.2$ & $33.0 \pm 14.6$ & $37.0 \pm 23.4$ & $0.26 \pm 0.42$ \\
\hline $\mathrm{J} 042216.27+265458.7$ & $41.7 \pm 11.7$ & $53.5 \pm 11.3$ & $-0.36 \pm 0.27$ \\
\hline J042242.40+264201.8 & $17.2 \pm 12.2$ & $>72.9 \pm 10.3$ & $-0.82 \pm 0.51$ \\
\hline $\mathrm{J} 042317.57+264228.7$ & $>37.3 \pm 24.4$ & $33.5 \pm 23.2$ & $1.11 \pm 0.62$ \\
\hline $\mathrm{J} 042426.28+261050.1$ & $55.2 \pm 13.9$ & $\ldots{ }^{a}$ & $<-0.36 \pm 0.14$ \\
\hline $\mathrm{J} 042634.81+260556.1$ & $55.0 \pm 10.6$ & $\ldots$ & $\ldots$ \\
\hline J042704.95+260429.3 & $51.9 \pm 14.2$ & $41.3 \pm 23.7$ & $0.01 \pm 0.53$ \\
\hline J042917.12+263023.3 & $29.6 \pm 13.5$ & $>62.3 \pm 16.0$ & $-0.49 \pm 0.61$ \\
\hline J042920.74+263353.4 & $65.8 \pm 9.9$ & $46.8 \pm 8.3$ & $0.65 \pm 0.26$ \\
\hline J042939.80+263222.9 & $51.2 \pm 16.6$ & $>27.2 \pm 29.1$ & $-1.05 \pm 0.49$ \\
\hline $\mathrm{J} 043055.31+260451.9$ & $13.0 \pm 26.4$ & $\ldots{ }^{a}$ & $<-0.05 \pm 0.14$ \\
\hline J043109.20+271045.3 & $57.9 \pm 7.7$ & $23.4 \pm 12.0$ & $1.02 \pm 0.26$ \\
\hline J043142.44+181101.5 & $>55.1 \pm 16.1$ & $\ldots$ a & $<-1.68 \pm 0.14$ \\
\hline J043237.91+242054.5 & $32.7 \pm 9.7$ & $65.4 \pm 10.1$ & $-0.41 \pm 0.41$ \\
\hline $\mathrm{J} 043323.81+260326.8$ & $.7 \pm 16.5$ & & \\
\hline $\mathrm{J} 043635.42+241324.2$ & $50.1 \pm 14.3$ & $>38.5 \pm 28.3$ & $-0.43 \pm 0.80$ \\
\hline $\mathrm{J} 043657.44+241835.1$ & $63.0 \pm 16.4$ & $\cdots$ & $\cdots$ \\
\hline J043816.85+252338.4 & $62.1 \pm 14.0$ & $\cdots$ & $\cdots$ \\
\hline $\mathrm{J} 043925.79+253825.3$ & $61.0 \pm 15.5$ & $>28.6 \pm 43.9$ & $0.85 \pm 1.07$ \\
\hline J044004.86+260421.2 & $27.4 \pm 19.9$ & $50.4 \pm 13.6$ & $0.08 \pm 0.45$ \\
\hline $\mathrm{J} 044022.18+260515.2$ & $\ldots{ }^{a}$ & $6.2 \pm 45.3$ & $>1.70 \pm 0.14$ \\
\hline J044042.98+255131.9 & $33.2 \pm 14.6$ & $1.3 \pm 26.5$ & $0.59 \pm 0.44$ \\
\hline J044123.47+245528.1 & $73.5 \pm 9.2$ & $\cdots$ & $\cdots$ \\
\hline $\mathrm{J} 044144.92+255815.3$ & $>69.0 \pm 12.8$ & $57.1 \pm 22.4$ & $-0.43 \pm 0.65$ \\
\hline J044210.59+252505.4 & $58.5 \pm 11.7$ & $58.0 \pm 19.9$ & $-0.68 \pm 0.61$ \\
\hline $\mathrm{J} 044233.26+251440.0$ & $48.4 \pm 13.3$ & $15.8 \pm 23.8$ & $0.44 \pm 0.41$ \\
\hline J044239.84+252306.4 & $60.3 \pm 14.6$ & $\cdots$ & $\cdots$ \\
\hline $\mathrm{J} 044246.20+251806.2$ & $81.5 \pm 6.5$ & $>28.6 \pm 39.8$ & $-0.54 \pm 0.84$ \\
\hline $\mathrm{J} 044256.30+251804.6$ & $27.7 \pm 23.5$ & $49.7 \pm 25.7$ & $0.61 \pm 0.77$ \\
\hline J045539.28+301627.2 & $24.4 \pm 9.3$ & $32.2 \pm 17.2$ & $0.49 \pm 0.39$ \\
\hline J045544.38+302455.1 & $62.7 \pm 9.0$ & $48.9 \pm 33.1$ & $-0.83 \pm 0.81$ \\
\hline J045547.39+303434.8 & $50.2 \pm 9.5$ & $30.5 \pm 23.8$ & $-0.83 \pm 0.47$ \\
\hline J045607.27+302728.2 & $37.9 \pm 15.7$ & $>30.2 \pm 23.3$ & $0.73 \pm 0.90$ \\
\hline
\end{tabular}

Young Stellar Object Candidates Based Only on Their Radio Properties

Note. ${ }^{\text {a }}$ Source not detected at three times the noise level on individual epochs, but detected on the image of the concatenated epochs.

most certainly extragalactic, while the nature of the other half is unclear. They might be previously unidentified YSOs, but (given the inherent uncertainties on the radio properties of different classes of active galactic nuclei) they could also be extragalactic background sources.

\subsection{Sources Identified as Both YSOs and Extragalactic}

We mentioned earlier that 60 of the detected radio sources are related to objects previously classified as YSOs. Eleven of these were also identified as extragalactic in the Sloan Digital Sky Survey (SDSS Adelman-McCarthy et al. 2011), they are listed 
Table 6

Radio Sources with Counterparts that are Clasified as Both YSOs and Extragalactic

\begin{tabular}{|c|c|c|c|c|}
\hline GBS-VLA Name & Other Names & $\begin{array}{c}\text { Variability } 4.5 \mathrm{GHz} \\
(\%)\end{array}$ & $\begin{array}{c}\text { Variability } 7.5 \mathrm{GHz} \\
(\%)\end{array}$ & Spectral Index \\
\hline J041833.38+283732.2 & 2MASS J04183343+2837321 & $30.1 \pm 28.6$ & $\cdots$ & $\cdots$ \\
\hline J041851.49+282026.1 & CoKu Tau 1 & $30.8 \pm 19.4$ & $23.9 \pm 22.6$ & $0.27 \pm 0.60$ \\
\hline J042200.70+265732.2 & 2MASS J04220069+2657324 & $31.8 \pm 32.3$ & $27.0 \pm 25.0$ & $0.54 \pm 0.61$ \\
\hline $\mathrm{J} 042202.20+265730.3$ & FS Tau A-B & $>51.6 \pm 17.3$ & $>52.8 \pm 18.7$ & $-0.29 \pm 0.33$ \\
\hline J042448.16+264315.9 & V1201 Tau & $45.1 \pm 7.7$ & $45.2 \pm 7.6$ & $-0.16 \pm 0.18$ \\
\hline J042702.56+260530.4 & DG Tau B & $25.1 \pm 15.1$ & $13.6 \pm 19.5$ & $0.39 \pm 0.34$ \\
\hline J042704.69+260615.8 & DG Tau & $11.1 \pm 10.2$ & $13.2 \pm 13.7$ & $0.10 \pm 0.25$ \\
\hline J042923.74+243259.8 & HARO 6-10 S & $13.7 \pm 10.9$ & $19.7 \pm 9.5$ & $0.30 \pm 0.20$ \\
\hline J043138.42+181357.3 & HL Tau & $13.9 \pm 17.6$ & $1.3 \pm 17.1$ & $0.68 \pm 0.36$ \\
\hline J043229.46+181400.2 & 2MASS J04322946+1814002 & $10.2 \pm 14.3$ & $17.2 \pm 38.9$ & $-0.25 \pm 0.71$ \\
\hline J043439.24+250100.9 & V1110 Tau & $>63.4 \pm 12.7$ & $>76.9 \pm 8.1$ & $-0.04 \pm 0.37$ \\
\hline
\end{tabular}

in Table 6. The radio properties of nine of these are consistent with being YSO (variability $>50 \%$ or $\alpha$ flat or positive).

One way to distinguish Galactic from extragalactic sources is by measuring their proper motions. As extragalactic objects are far away we do not expect to measure proper motions in them. On the other hand, members of the Taurus-Auriga region

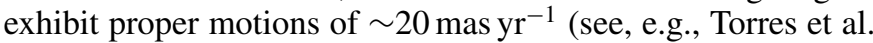
2009). The proper motions of two of the sources in Table 6 , have been measured at radio wavelengths, DG Tau (Rodríguez et al. 2012a) and DG Tau B (Rodríguez et al. 2012b), and were found to be consistent with those measured to other members in Taurus-Auriga.

By combining the position for Haro 6-10 S reported by Reipurth et al. (2004), with those obtained by us, we can roughly estimate the proper motions of this source. We obtained $\mu_{\alpha} \cos \delta=16.1 \pm 7.9$ mas yr$^{-1}$ and $\mu_{\delta}=-33.8 \pm$

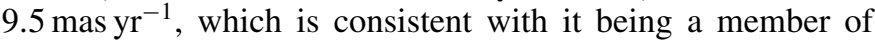
Taurus-Auriga.

On the other hand, two of the sources in Table 6 (GBS-VLA J043229.46+181400.2 and GBS-VLA J041833.38+283732.2) have radio properties (low variability with negative spectral index) more typical of extragalactic objects than of YSOs.

GBS-VLA J043229.46+181400.2 has a radio flux of order $\sim 60 \mathrm{mJy}$ which is large compared with most of the other YSOs in Taurus-Auriga. It was classified as a Class II YSO by Gutermuth et al. (2009) based on its infrared properties. We looked in the literature for a good determination of its position at radio frequencies, but we did not find any. Thus, we looked for a high resolution, good sensitivity observation in the VLA archive. We found one observation obtained in 1995 September as part of the project BB0051. After a standard calibration of these data we measured the position for this source to be R.A. $=04^{\mathrm{h}} 32^{\mathrm{m}} 29^{\mathrm{s}} .460$ and decl. $=18^{\circ} 14^{\prime} 00^{\prime \prime} .19$, with an error in position of $\sim 0^{\prime \prime} .01$. Combining this with our own observations, we obtain a rough estimate of the proper motions for this source: $\mu_{\alpha} \cos \delta=4.6 \pm 3.3 \mathrm{mas} \mathrm{yr}^{-1}$ and $\mu_{\delta}=5.2 \pm 3.9$ mas yr$^{-1}$. These results are consistent with no motion at all at the $1.4 \times \sigma_{\mu}$ level. This strongly suggests that GBS-VLA J043229.46+181400.3 is a background source rather than a YSO, and we will not consider it as a YSO in the rest of the paper.

The remaining source, GBS-VLA J041833.38+283732.2, was only observed in the $4.5 \mathrm{GHz}$ sub-band and does not show significant levels of variability. At infrared wavelengths it was classified as a Class I YSO, and low variability is expected for most of the stars in this evolutionary status (e.g., Dzib et al. 2013a). Also, we note that all the YSOs in the evolutionary Class I that are detected by the SDSS, are classified as extragalactic by them, suggesting a bias in the SDSS identification criteria. Thus, we will consider it as a YSO. In summary, we consider that 10 of the 11 sources in Table 6 are counterparts of YSOs, and that 59 of the 60 sources previously classified as YSOs are in fact YSOs.

\subsection{General Radio Properties of the YSO Population}

Sources identified as YSOs are listed in Table 7. From their radio properties, 33 objects show at least one clear indication that their emission is non-thermal in nature (high variability, circular polarized emission or negative spectral index). Another 22 objects are consistent with their emission being thermal free-free emission (positive spectral index and low variability). From the remaining sources three are non-variable, do not show signs of circular polarization, but they show a negative spectral index, that given the involved errors, the nature of their emissions remains uncertain. Finally, we do not have enough information to favor a thermal or non-thermal emission mechanism for GBS-VLA J041833.38+283732.2. Our results show that a significant population of the YSOs in Taurus-Auriga $(56 \%)$ have radio emission that is non-thermal in nature. These stars can be used for Very Long Baseline Interferometry (VLBI) astrometric observations (e.g., Dzib et al. 2010, 2011; Torres et al. 2012).

The evolutionary status is known for 54 of the 59 YSOs detected in this radio survey. In addition, 53 have a known spectral type. Thus, we can compare their radio properties to their evolutionary status and spectral type.

We have found previously that, on average, more evolved stars show radio properties that resemble a non-thermal origin (i.e., more variable and with more negative spectral indices, Dzib et al. 2013a; Ortiz-León et al. 2014; Kounkel et al. 2014). This is in agreement with the idea that more evolved sources have already shed away most of their surrounding material and that we are detecting the non-thermal emission from their coronae instead of the thermal emission from the surrounding material (see Dzib et al. 2013a for a discussion). From Figures 2 and 3 (where we have also plotted our previous result in Ophiuchus) we see that the YSOs in Taurus-Auriga follow the same tendency. Note, however, that at each of the evolutionary stages we found both thermal and non-thermal radio emitters (see also Table 7) as also noted in Ophiuchus (Dzib et al. 2013a), Serpens and W40 (Ortiz-León et al. 2014) and Orion (Kounkel et al. 2014). The weighted 
Table 7

Young Stellar Objects Detected in the Radio Observations

\begin{tabular}{|c|c|c|c|c|c|c|c|c|}
\hline GBS-VLA Name & $\begin{array}{c}\text { Spectral } \\
\text { Type }\end{array}$ & Reference & $\begin{array}{c}\text { SED } \\
\text { Clasification }\end{array}$ & Reference & Var. & Pol. & $\alpha$ & X-Ray \\
\hline J040349.35+261051.8 & M2 & 1 & Class III & 1,2 & $\mathrm{~N}$ & $\mathrm{~N}$ & $\mathrm{~F}$ & $\mathrm{Y}$ \\
\hline $\mathrm{J} 040443.07+261856.3$ & K4 & 3 & Class I & $1,2,3$ & $\mathrm{~N}$ & $\mathrm{~N}$ & $\mathrm{P}$ & $\mathrm{Y}$ \\
\hline J041314.16+281910.3 & M4 & 4 & Class III & 1,2 & $\mathrm{Y}$ & $\mathrm{N}$ & $\mathrm{N}$ & $\mathrm{Y}$ \\
\hline J041327.23+281624.4 & M0 & 4 & Class III & 1,2 & $\mathrm{Y}$ & $\mathrm{Y}$ & $\mathrm{N}$ & $\mathrm{Y}$ \\
\hline J041354.72+281132.6 & K0-M3 & 5 & Class I & 1,3 & $\mathrm{Y}$ & $\mathrm{N}$ & $\mathrm{P}$ & $\mathrm{Y}$ \\
\hline J041412.93+281211.9 & K3 & 6 & WTTS/Class II & $1,2,7$ & $\mathrm{Y}$ & $\mathrm{N}$ & $\mathrm{P}$ & $\mathrm{Y}$ \\
\hline J041426.41+280559.4 & M2.5/2.5 & 4 & Class II & 8 & $\mathrm{Y}$ & $\mathrm{N}$ & $\mathrm{F}$ & $\mathrm{Y}$ \\
\hline $\mathrm{J} 041430.55+280514.4$ & K7 & 4 & Class II & 9 & $\mathrm{~N}$ & $\mathrm{~N}$ & $\mathrm{P}$ & $\mathrm{Y}$ \\
\hline $\mathrm{J} 041448.00+275234.4$ & M1 & 6,10 & $\ldots$ & $\ldots$ & $\mathrm{Y}$ & $\mathrm{N}$ & $\mathrm{P}$ & $\mathrm{Y}$ \\
\hline J041628.11+280735.4 & K7V & 11 & $\cdots$ & $\cdots$ & $\mathrm{Y}$ & $\mathrm{Y}$ & $\mathrm{N}$ & $\mathrm{N}$ \\
\hline J041829.10+282618.8 & M1 & 4 & $\cdots$ & $\cdots$ & $\mathrm{N}$ & $\mathrm{N}$ & $\mathrm{N}$ & $\mathrm{Y}$ \\
\hline J041831.12+282715.9 & K4 & 12 & Class III & 1,2 & $\mathrm{~N}$ & $\mathrm{Y}$ & $\mathrm{N}$ & $\mathrm{Y}$ \\
\hline J041833.38+283732.2 & $\cdots$ & $\cdots$ & Class I & 8 & $\mathrm{~N}$ & - & - & $\mathrm{N}$ \\
\hline J041834.45+283029.9 & M0 & 4 & Class II & 8 & $\mathrm{~N}$ & $\mathrm{~N}$ & $\mathrm{~F}$ & $\mathrm{Y}$ \\
\hline J041840.62+281915.3 & B8 & 13 & Class II & 1,2 & $\mathrm{Y}$ & $\mathrm{Y}$ & $\mathrm{P}$ & $\mathrm{Y}$ \\
\hline $\mathrm{J} 041847.04+282007.2$ & K7 & 4 & Class III & 1,2 & $\mathrm{Y}$ & $\mathrm{Y}$ & $\mathrm{P}$ & $\mathrm{Y}$ \\
\hline $\mathrm{J} 041851.49+282026.1$ & K7 & 3 & Class I & $1,2,3$ & $\mathrm{~N}$ & $\mathrm{~N}$ & $\mathrm{P}$ & $\mathrm{N}$ \\
\hline J041915.84+290626.6 & K7 & 14 & Class II & 1,2 & $\mathrm{~N}$ & $\mathrm{~N}$ & $\mathrm{P}$ & $\mathrm{Y}$ \\
\hline J041926.27+282614.0 & K7 & 4,6 & Class III & 1,2 & $\mathrm{Y}$ & $\mathrm{N}$ & $\mathrm{N}$ & $\mathrm{Y}$ \\
\hline J041941.28+274947.9 & M0 & 6,10 & $\cdots$ & $\cdots$ & $\mathrm{Y}$ & $\mathrm{Y}$ & $\mathrm{P}$ & $\mathrm{Y}$ \\
\hline J042157.41+282635.3 & $\mathrm{K} 1$ & 1 & Class II & 1,2 & $\mathrm{~N}$ & $\mathrm{~N}$ & $\mathrm{P}$ & Y \\
\hline J042159.43+193205.7 & K0 & 1 & Class II & $1,2,3$ & $\mathrm{Y}$ & $\mathrm{Y}$ & $\mathrm{N}$ & $\mathrm{Y}$ \\
\hline $\mathrm{J} 042200.70+265732.2$ & $\ldots$ & $\cdots$ & Class I & 15 & $\mathrm{~N}$ & $\mathrm{~N}$ & $\mathrm{P}$ & $\mathrm{N}$ \\
\hline $\mathrm{J} 042202.20+265730.3$ & M0-M3.5 & 3 & Class II & 3 & $\mathrm{Y}$ & $\mathrm{N}$ & $\mathrm{N}$ & $\mathrm{Y}$ \\
\hline J042203.15+282538.8 & M3 & 1 & Class III & 1,2 & $\mathrm{~N}$ & $\mathrm{Y}$ & $\mathrm{F}$ & $\mathrm{Y}$ \\
\hline J042204.97+193448.3 & M4.5 & 16 & WTTS & 16 & $\mathrm{Y}$ & $\mathrm{N}$ & $\mathrm{F}$ & $\mathrm{Y}$ \\
\hline $\mathrm{J} 042448.16+264315.9$ & K1 & 16 & WTTS & 16 & $\mathrm{~N}$ & $\mathrm{~N}$ & $\mathrm{~F}$ & $\mathrm{Y}$ \\
\hline $\mathrm{J} 042449.05+264310.2$ & K0 & 16 & Class III & 17 & $\mathrm{Y}$ & $\mathrm{N}$ & $\mathrm{F}$ & $\mathrm{N}$ \\
\hline $\mathrm{J} 042517.70+261750.2$ & K7 & 15 & Class III & 15 & $\mathrm{Y}$ & $\mathrm{N}$ & $\mathrm{P}$ & $\mathrm{Y}$ \\
\hline J042702.56+260530.4 & $\ldots$ & $\cdots$ & Class II & 3 & $\mathrm{~N}$ & $\mathrm{~N}$ & $\mathrm{P}$ & $\mathrm{N}$ \\
\hline $\mathrm{J} 042704.69+260615.8$ & K6 & 4 & Class II & 1,3 & $\mathrm{~N}$ & $\mathrm{~N}$ & $\mathrm{~F}$ & $\mathrm{Y}$ \\
\hline J042920.70+263340.2 & M4 & 4 & Class III & 1,2 & $\mathrm{~N}$ & $\mathrm{~N}$ & $\mathrm{~N}$ & $\mathrm{Y}$ \\
\hline J042923.74+243259.8 & K3-7 & 1,3 & Class I & $1,2,3$ & $\mathrm{~N}$ & $\mathrm{~N}$ & $\mathrm{P}$ & $\mathrm{N}$ \\
\hline J042923.74+243301.3 & K7-M2 & 18 & $\cdots$ & $\cdots$ & $\mathrm{N}$ & $\mathrm{N}$ & $\mathrm{P}$ & $\mathrm{N}$ \\
\hline $\mathrm{J} 042942.47+263249.0$ & M0 & 4 & Class III & 1 & $\mathrm{Y}$ & $\mathrm{N}$ & $\mathrm{N}$ & $\mathrm{Y}$ \\
\hline J043114.45+271017.6 & M0.5 & 15 & Class III & 15 & $\mathrm{Y}$ & $\mathrm{N}$ & $\mathrm{P}$ & Y \\
\hline J043138.42+181357.3 & K5 & 3 & Class I & $2,3,8$ & $\mathrm{~N}$ & - & $\mathrm{P}$ & $\mathrm{Y}$ \\
\hline $\mathrm{J} 043140.09+181356.7$ & $\mathrm{M} 2 / 3.5$ & 19 & Class II & 1,3 & $\mathrm{Y}$ & $\mathrm{Y}$ & $\mathrm{N}$ & Y \\
\hline J043144.49+180831.6 & $\cdots$ & $\cdots$ & Class I & $1,2,3$ & $\mathrm{~N}$ & $\mathrm{~N}$ & $\mathrm{P}$ & $\mathrm{Y}$ \\
\hline $\mathrm{J} 043158.46+254329.8$ & M5.5 & 4 & Class III & 1 & $\mathrm{~N}$ & $\mathrm{~N}$ & $\mathrm{P}$ & $\mathrm{Y}$ \\
\hline J043214.58+182014.6 & K7 & 4 & Class III & 1,2 & $\mathrm{Y}$ & $\mathrm{Y}$ & $\mathrm{N}$ & $\mathrm{Y}$ \\
\hline J043215.42+242859.3 & M0 & 3 & Class II & 3 & $\mathrm{~N}$ & $\mathrm{~N}$ & $\mathrm{P}$ & $\mathrm{Y}$ \\
\hline J043215.86+180138.5 & K7 & 4 & Class III & 1,2 & $\mathrm{~N}$ & $\mathrm{~N}$ & $\mathrm{~F}$ & $\mathrm{Y}$ \\
\hline $\mathrm{J} 043232.07+225726.3$ & K0-M4 & 5 & Class I & $1,3,15$ & $\mathrm{Y}$ & $\mathrm{N}$ & $\mathrm{N}$ & $\mathrm{Y}$ \\
\hline J043242.82+255231.2 & $\mathrm{M} 2.0 \mathrm{e}+\mathrm{M} 3.0 \mathrm{e}$ & 19 & Class II & 15 & $\mathrm{~N}$ & $\mathrm{~N}$ & $\mathrm{P}$ & $\mathrm{Y}$ \\
\hline $\mathrm{J} 043243.07+255230.7$ & $\mathrm{M} 1 \mathrm{~V}+\mathrm{M} 4 \mathrm{~V}$ & 20 & Class II & 15 & $\mathrm{Y}$ & $\mathrm{N}$ & $\mathrm{P}$ & $\mathrm{N}$ \\
\hline J043306.62+240954.8 & K7/M3 & 15 & Class II & 15 & $\mathrm{Y}$ & $\mathrm{N}$ & $\mathrm{F}$ & $\mathrm{Y}$ \\
\hline $\mathrm{J} 043310.04+243343.1$ & K7 & 4 & Class III & 1,2 & $\mathrm{Y}$ & $\mathrm{N}$ & $\mathrm{P}$ & $\mathrm{Y}$ \\
\hline J043439.24+250100.9 & K0 & 15 & Class III & 15 & $\mathrm{Y}$ & $\mathrm{N}$ & $\mathrm{F}$ & Y \\
\hline J043520.92+225424.0 & K7 & 4,15 & Class III & $1,2,15$ & $\mathrm{Y}$ & $\mathrm{N}$ & $\mathrm{P}$ & Y \\
\hline $\mathrm{J} 043540.95+241108.6$ & M1 & 4,15 & Class II & $1,2,15$ & $\mathrm{Y}$ & $\mathrm{N}$ & $\mathrm{P}$ & $\mathrm{Y}$ \\
\hline J043553.52+225408.9 & K7 & 4 & Class III & 1,2 & $\mathrm{Y}$ & $\mathrm{N}$ & $\mathrm{N}$ & $\mathrm{Y}$ \\
\hline J043935.20+254144.3 & $\cdots$ & $\cdots$ & Class I & $1,2,3$ & $\mathrm{Y}$ & $\mathrm{N}$ & $\mathrm{P}$ & $\mathrm{Y}$ \\
\hline J043953.86+260309.7 & $\cdots$ & $\cdots$ & Class 0 & 21 & $\mathrm{~N}$ & $\mathrm{~N}$ & $\mathrm{~F}$ & $\mathrm{~N}$ \\
\hline $\mathrm{J} 043955.75+254501.7$ & K4 & 3 & Class II & $1,2,3$ & $\mathrm{~N}$ & $\mathrm{~N}$ & $\mathrm{P}$ & $\mathrm{Y}$ \\
\hline $\mathrm{J} 044205.49+252256.0$ & K7 & 15 & Class III & 15 & $\mathrm{Y}$ & $\mathrm{N}$ & $\mathrm{N}$ & $\mathrm{Y}$ \\
\hline $\mathrm{J} 044207.32+252303.0$ & M1 & 15 & Class III & 15 & $\mathrm{~N}$ & $\mathrm{~N}$ & $\mathrm{~N}$ & $\mathrm{Y}$ \\
\hline $\mathrm{J} 045536.97+301754.8$ & K0 & 22 & WTTS & 22 & $\mathrm{Y}$ & $\mathrm{N}$ & $\mathrm{F}$ & $\mathrm{Y}$ \\
\hline $\mathrm{J} 045545.85+303304.0$ & A0 & 23 & Class II & 1,2 & $\mathrm{~N}$ & $\mathrm{~N}$ & $\mathrm{P}$ & $\mathrm{Y}$ \\
\hline
\end{tabular}

Notes. Var. $=\mathrm{Y}$ when the source variability is higher than $50 \%$ in at least one frequency; $\mathrm{N}$ when it is lower. Pol. $=\mathrm{Y}$ when circular polarization is detected and $\mathrm{N}$ when it is not. Sources in the outer fields for which the polarization could not be assessed are shown as "-." $\alpha$ refers to the spectral index, and is given as $\mathrm{P}$ (for positive) when it is higher than 0.2 ; $\mathrm{F}$ (for flat) when it is between -0.2 and +0.2 , and $\mathrm{N}$ (for negative) when it is lower than -0.2 . $\mathrm{X}$-ray $=\mathrm{Y}$ when there is an $\mathrm{X}$-ray flux reported in the literature, $\mathrm{N}$ when it is not.

References. 1 = Kenyon \& Hartmann (1995); $2=$ Andrews \& Williams (2005); 3= White \& Hillenbrand (2004); $4=$ Briceño et al. (2002); $5=$ Connelley \& Greene (2010); 6 = Riviere-Marichalar et al. (2012); 7 = Hartigan et al. (1994); $8=$ Gutermuth et al. (2009); $9=$ Hartmann (2002); $10=$ Voges et al. (1999); 11 = Devor et al. (2008); 12 = White \& Ghez (2001); 13 = Manoj et al. (2006); 14 = Donati et al. (2008); $15=$ Rebull et al. (2010); 16 = Martín \& Magazzù (1999); $17=$ Wahhaj et al. (2010); 18 = Doppmann et al. (2008); $19=$ Hartigan \& Kenyon (2003); $20=$ Prato et al. (2002); $21=$ Chini et al. (2001); $22=$ Furlan et al. (2011) and $23=$ Hernández et al. (2004). 


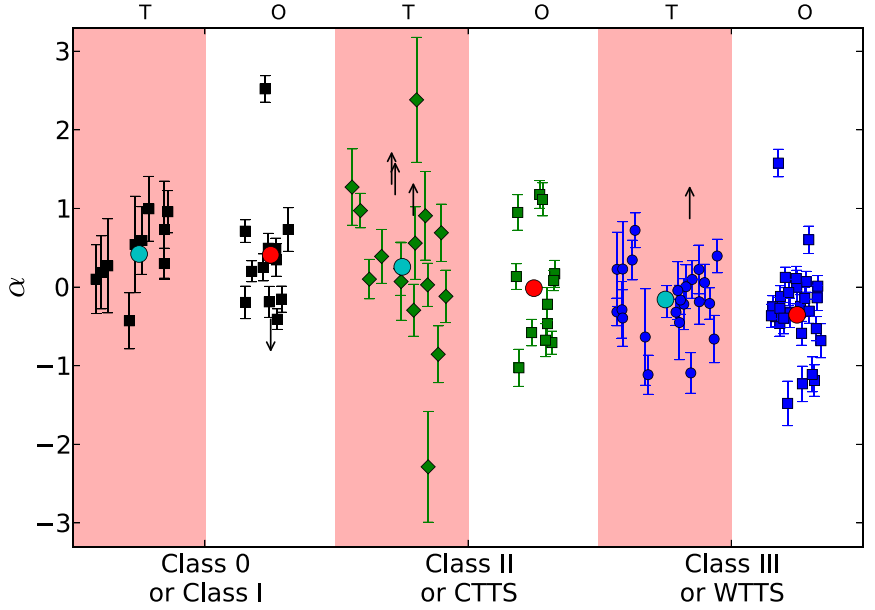

Figure 2. Spectral index as a function of the YSO evolutionary stages for YSOs in Taurus-Auriga (labeled in the top with a T) and Ophiuchus (labeled in the top with an O). The individual sources are shown with their error bars, and the turquoise and red circles indicate the mean spectral index for each category in Taurus-Auriga and Ophiuchus, respectively. Ophiuchus data from Dzib et al. (2013a).

average flux density seems also to be slightly higher for the more evolved YSOs (see Figure 4), but it is not as dramatic as in the Ophiuchus case (Dzib et al. 2013a; Figure 4).

Finally, Figure 5 shows the flux density of the YSOs as a function of their spectral type. As in the Ophiuchus case, most of the YSOs sources detected at radio frequencies have a spectral type $\mathrm{M}$ and $\mathrm{K}$, there are no detected sources of types $\mathrm{G}$ and $\mathrm{F}$, and only one A-type and one B-type detection. In part, this presumably reflects the fact that the Taurus-Auriga star-forming region contains mainly low-mass stars. Contrary to the results in
Ophiuchus, in Taurus-Auriga the averaged radio flux density for the low-mass members seems to be larger than the intermediatemass members. We discuss the peculiarities of these stars in the next section.

\subsection{The Radio-X-Ray Relation}

An interesting result that we have been noticing in our VLA surveys is the relation between the radio emission of YSOs compared to their previously reported X-ray emission. The recent results indicate that YSOs follow the so-called Güdel-Benz relation (Guedel \& Benz 1993 and Benz \& Guedel 1994) for magnetically active stars. The relation has the general form (Benz \& Guedel 1994):

$$
\frac{L_{X}}{L_{R, v}}=\kappa \cdot 10^{15.5 \pm 0.5}[\mathrm{~Hz}] .
$$

The value of $\kappa$ varies for different kind of stars and fulfills $\kappa \leqslant 1$. Particularly, we proposed a $\kappa=0.03$ for YSOs, based just in the positions on the $L_{X}-L_{R}$ diagram of YSOs in the Ophiuchus star-forming regions (Dzib et al. 2013a), and also following the previous results obtained by Gagné et al. (2004) and Forbrich et al. (2010).

We searched for X-ray counterparts of the YSOs detected in Taurus-Auriga (see also Table 4) and plot them against the radio luminosities in Figure 6 with blue symbols. In Figure 6 we also plotted the results obtained in Ophiuchus (green symbols, from Dzib et al. 2013a), Serpens-W40 (red symbols; from Ortiz-León et al. 2014), and Orion (yellow symbols; from Kounkel et al. 2014). Most of the YSOs seem to follow, with $\sim 1$ dex of dispersion, the Güdel-Benz relation with $\kappa=0.03$. There are, however, some clear exceptions in the Orion region, where some sources are apparently X-ray underluminous by more than
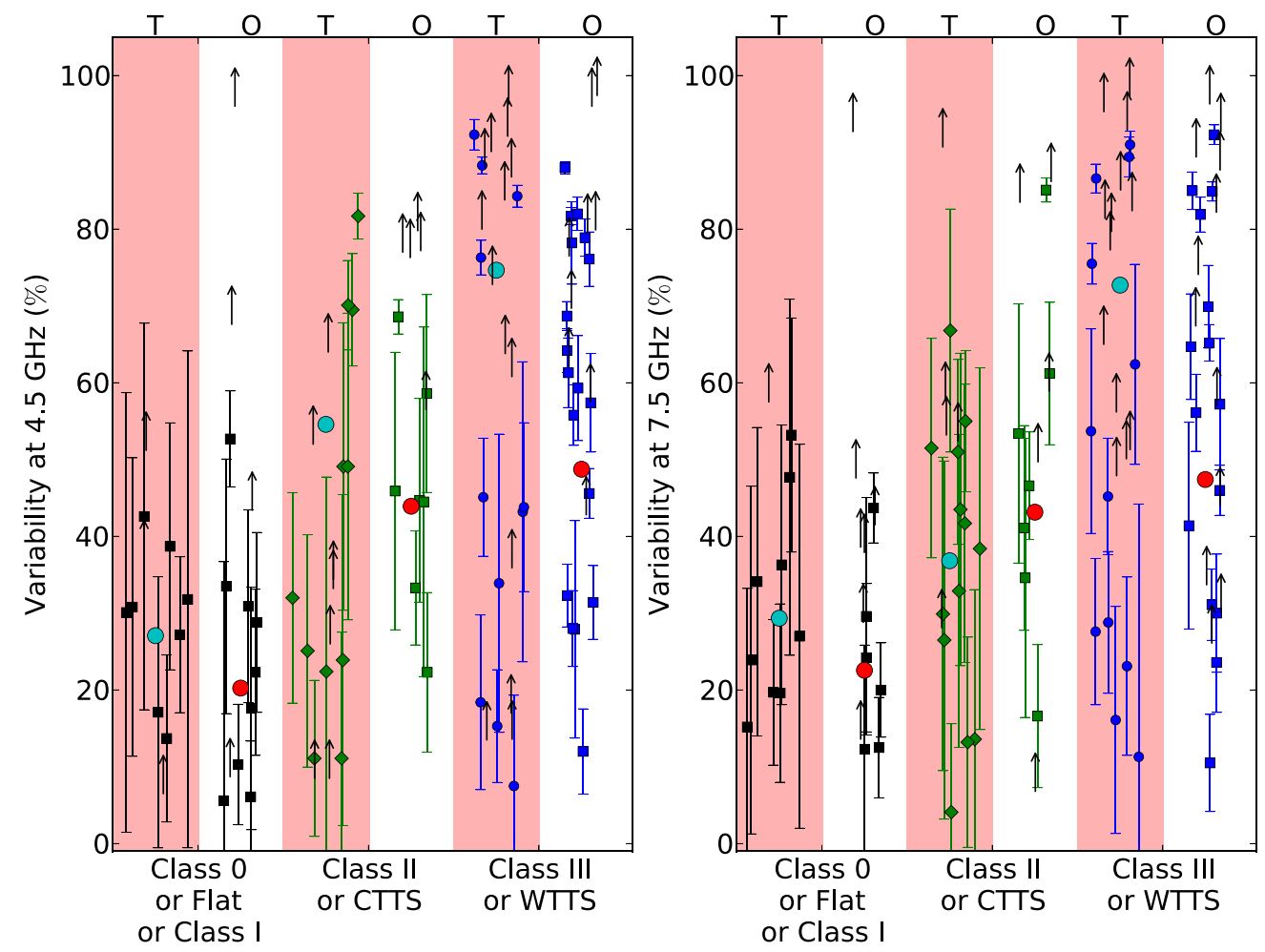

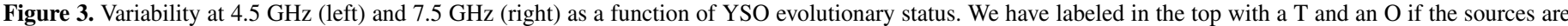

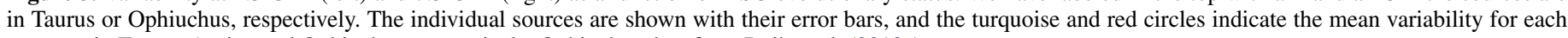
category in Taurus-Auriga and Ophiuchus, respectively. Ophiuchus data from Dzib et al. (2013a). 

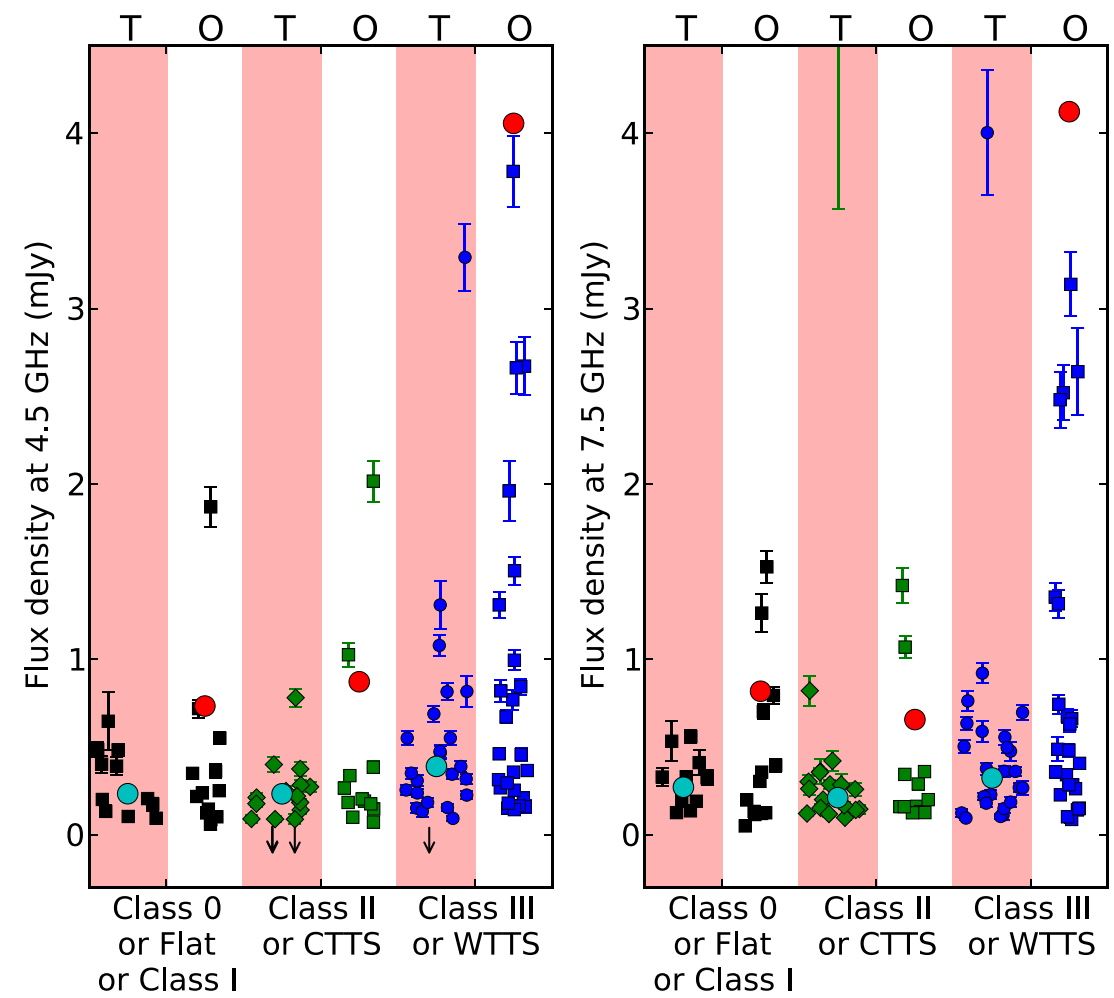

Figure 4. Radio flux at $4.5 \mathrm{GHz}$ (left) and $7.5 \mathrm{GHz}$ (right) as a function of YSO evolutionary status. We have labeled in the top with a $\mathrm{T}$ and an $\mathrm{O}$ if the sources are in Taurus or Ophiuchus, respectively. As in the previous figures, the individual sources are shown with their error bars, and the turquoise and red circles indicate the mean flux for each category in Taurus-Auriga and Ophiuchus, respectively. Ophiuchus data from Dzib et al. (2013a). We limit the figure to a flux of $4.5 \mathrm{mJy}$ to see the slope between the mean fluxes in Taurus.
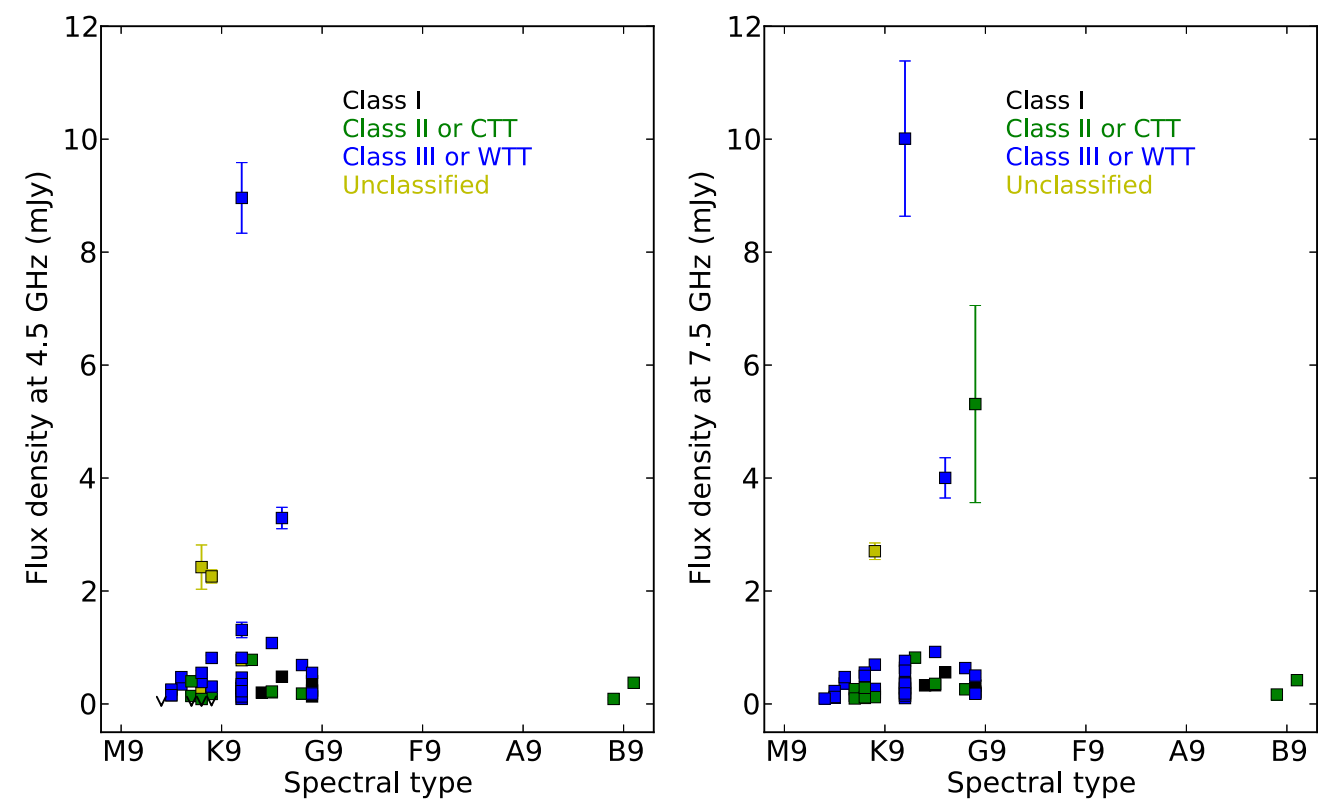

Figure 5. Radio flux at $7.5 \mathrm{GHz}$ (left) and $4.5 \mathrm{GHz}$ (right) as a function of YSO spectral type. Colors indicate the evolutionary class of the object as listed at the top-right of the diagrams.

two orders of magnitude. We noticed that this trend does not depend on the evolutionary classification of the YSOs (squares for Class I/FS, rhombus for Class II/CTTS, circles for Class III/ WTTS, and pentagons for unclassified sources in Figure 6) or if the radio emission is most likely to be thermal (open symbols) or non-thermal (filled symbols). Dzib et al. (2013b) has discussed that even when the gyrosynchrotron radio emission is hidden by free-free radio emission in upper layers of circumstellar material, the X-ray emission produced in the plasma of the corona could still be observed. Even more interesting is that, as noticed by Dzib et al. (2013b), independent of the dominant radio emission mechanism the YSOs seem to fit the Güdel-Benz relation, meaning that both radio emission mechanisms have similar levels of flux densities. 

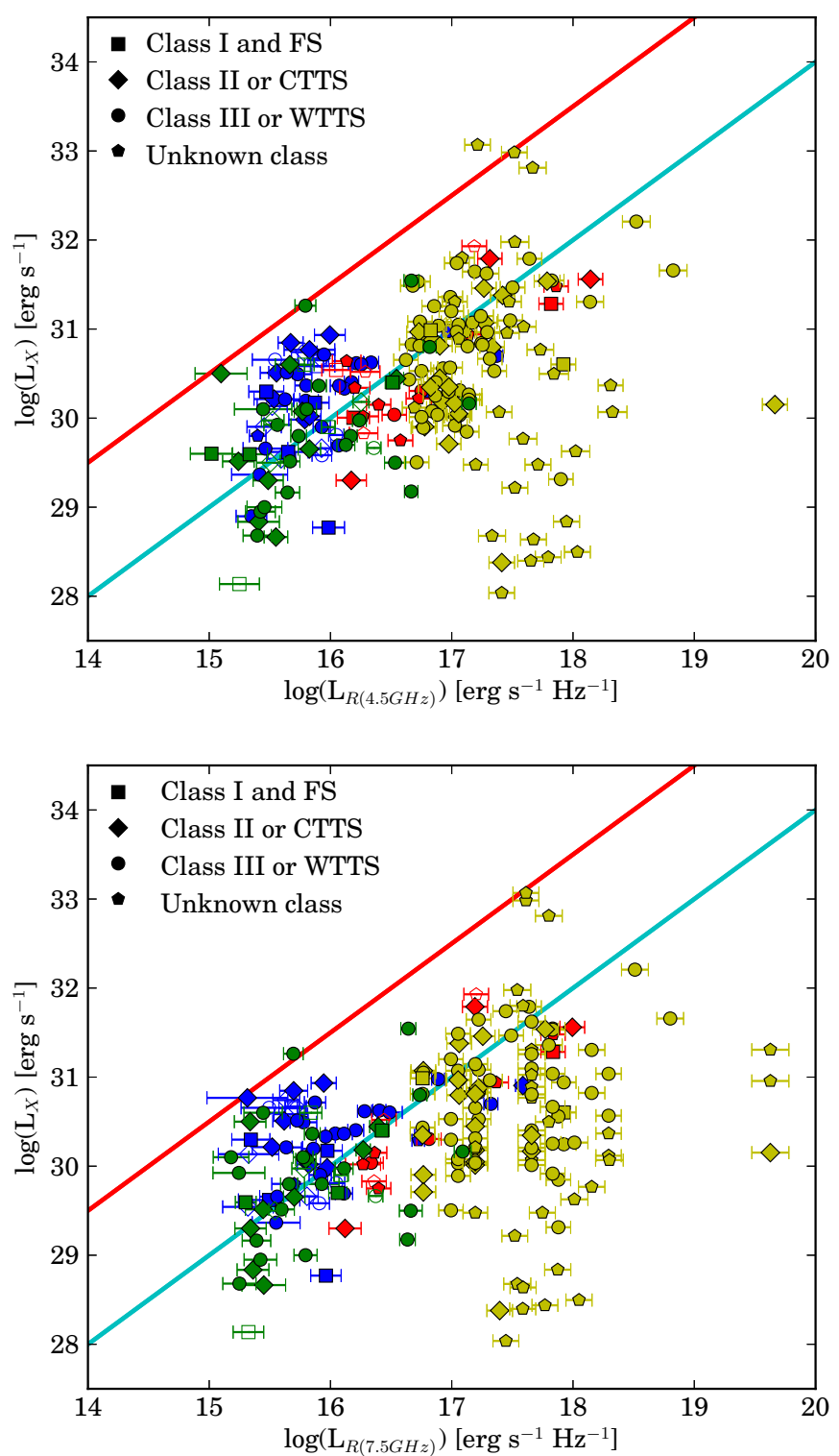

Figure 6. X-ray luminosity as a function of radio luminosity. The red line corresponds to the Güdel-Benz relation with $\kappa=1$. The blue line correspond to the Güdel-Benz relation but with $\kappa=0.03$. Symbols indicate the evolutionary status of the object as explained at the top-left of the diagram. Colors indicates YSOs in different star-forming regions: Taurus (blue, this work), Ophiuchus (green, Dzib et al. 2013a), Serpens-W40 (red, Ortiz-León et al. 2014) and Orion (yellow, Kounkel et al. 2014). Open symbols indicate sources whose radio emission is thermal and solid symbols indicates non-thermal radio sources.

\section{COMMENTS ON SOME INDIVIDUAL SOURCES}

\subsection{Non-thermal Class I Stars}

Newly born low-mass stars (between $0.08 M_{\odot}$ to $3.0 M_{\odot}$ ) are magnetically active and could produce significant amount of gyrosynchrotron radio emission from their coronae. In the very young stars (i.e., Class I) this radio emission is expected to be hidden by the optically thick free-free radio emission from the surrounding material. There are a few cases, however, where non-thermal radio emission has been detected from Class I stars (e.g., Feigelson et al. 1998; Forbrich et al. 2007; Dzib et al. 2010, 2013a and Deller et al. 2013). Geometric effects or abnormally early removal of the surrounding material (e.g., in a tight binary system) have been invoked to explain these detections (e.g., Forbrich et al. 2007; Dzib et al. 2010). Thus, it is important to document more cases in order to identify which is the dominant mechanism that allows the detection of coronal emission in very young YSOs. Three Class I stars in our sample have properties that indicate their radio emission could be of a nonthermal nature: GBS-VLA J041354.72+281132.6, GBS-VLA $J 043232.07+225726.3$ and GBS-VLA J043935.20+254144.3 are Class I stars that are highly variable, which is an indication that their emission has a non-thermal origin. In the case of $G B S$-VLA J043232.07+225726.3 this is also supported by the negative spectral index. The non-thermal origin of the emission from these very young stars must be confirmed using other techniques, e.g., VLBI.

\subsection{Low Variability Non-thermal Radio Emission}

Non-thermal radio emission from YSOs often exhibits high levels of variability $(>50 \%)$ associated with magnetic activity of the star (Feigelson \& Montmerle 1999). There are, however, some exceptions to this. The $6 M_{\odot}$ star $\mathrm{S} 1$ in the Ophiuchus core shows only modest levels of variability during a series of VLBI observations made by Loinard et al. (2008). This suggests a different origin with more organized magnetic fields. For a better understanding of this behavior, further studies must be carried with more objects. In the present report we detected two other sources which are likely non-thermal because they have detectable circular polarization, but which are only moderately variable.

GBS-VLA J041831.12+282715.9 = V 410 Tau, is a K4 star that shows variabilities below $30 \%$ during the three observed epochs. Circular polarized emission was found at a level of $8.7 \%(\mathrm{R})$ in the $7.5 \mathrm{GHz}$ sub-band. Also, it shows a negative spectral index $(\alpha=-0.32 \pm 0.17)$ which supports the nonthermal origin. Carroll et al. (2012) found that the magnetic topology in the visible pole of V 410 is dominated by a bipolar structure. These authors also argue that the absence of a radiative core and non-detection of differential rotation support the idea that a classical dynamo is not operating in V 410 Tau.

GBS-VLA J042203.15+282538.8 is an M3 star with measured variability below $16 \%$. Its spectral index between the observed sub-bands is flat $(\alpha=0.00 \pm 0.28)$. Circular polarization is detected in both sub-bands at levels of $21.3 \%(\mathrm{~L})$ and $30.8 \%$ (L) for $4.5 \mathrm{GHz}$ and $7.5 \mathrm{GHz}$, respectively. These levels of circular polarization strongly suggest a non-thermal origin.

\subsection{Sources with Large Positive Spectral Indices}

Only two sources have spectral indices that are consistent with large positive values $(\sim 2)$. These sources are GBS-VLA J043540.95+241108.6 (with spectral index $2.38 \pm 0.79$ ) and GBS-VLA J044022.18+260515.2 (with spectral index $>1.70 \pm 0.14)$. The first source is associated with $\mathrm{CoKu}$ Tau 3, a well known Class II YSO and we attribute the spectral index to the presence of optically thick gyrosynchrotron emission. The second source, GBS-VLA J044022.18+260515.2, has been tentatively classified by us as a YSO candidate on the basis of its positive spectral index. An alternative interpretation is that it is a HFP. These are compact extragalactic radio sources with welldefined peaks in their radio spectra above $5 \mathrm{GHz}$ and positive spectral indices at frequencies below their peak. Most of them are believed to be high redshift quasars (Orienti \& Dallacasa 2014). The case of a possible HFP observed in projection in the star-forming region M17 is discussed by Rodríguez et al. (2014a). 


\subsection{The Very Low-mass Star GBS-VLA J043158.46+254329.8}

Dzib et al. (2013a) show that the detection of YSOs near and beyond the brown dwarf boundary at distances farther than $100 \mathrm{pc}$ is possible. They reported the radio emission of four very low-mass stars in the Ophiuchus core. Three of them show indications that the origin of their radio emission is non-thermal.

GBS-VLA J043158.46+254329.8 is a Class III YSO (Kenyon \& Hartmann 1995), with a mass of $0.19 M_{\odot}$ and an M5.5 spectral type (Briceño et al. 2002). It was not detected in the $4.5 \mathrm{GHz}$ subband on individual epochs at levels of $100 \mu \mathrm{Jy}$ nor in the image of the three concatenated epochs at a level of $63 \mu \mathrm{Jy}$. On the other hand, in the $7.5 \mathrm{GHz}$ sub-band it was not detected in the first two epochs at levels of $80 \mu \mathrm{Jy}$, but in the third epoch it was detected with a total flux density of $165 \pm 28 \mu \mathrm{Jy}$. This variation $(>45 \%)$ is just slightly below our adopted limit of high variability and suggests that GBS-VLA J043158.46+254329.8 could also be a very low-mass star with non-thermal radio emission.

\subsection{Non-thermal Radio Emission from the Close Binary Elias $1=$ GBS-VLA J041840.62+281915.3}

Elias 1 is a well known Herbig AeBe $(\mathrm{HAeBe})^{12}$ star in the Taurus-Auriga cloud (Elias 1978). It has two companions, a $\mathrm{T}$ Tauri star at $4^{\prime \prime}$ to its northeast (Leinert et al. 1997) and a close (50 mas) low-mass companion (Smith et al. 2005). It is variable at X-rays frequencies, and an X-ray flare was reported by Giardino et al. (2004). This is an indication that a corona is acting in at least one of the two components of the close binary. At radio frequencies, we detected a source related with the close binary (GBS J041840.62+281915.3). It is a highly variable star in both of the observed sub-bands $(69.5 \% \pm 16.7 \%$ and $51.5 \% \pm 15.2 \%$ at $4.5 \mathrm{GHz}$ and $7.5 \mathrm{GHz}$, respectively). This is another indication that a corona is involved.

The standard theory of intermediate-mass stellar evolution predicts that during the pre-main sequence (and, indeed, the main sequence), these stars of relative large masses are fully radiative. As a consequence, they are not expected to drive a dynamo, and should not have strong surface magnetic fields. Thus, they should not maintain a corona. X-ray observations have shown that some HAeBe stars have indeed signs that they can host coronae that resemble those of T Tauri stars (Damiani et al. 1994; Zinnecker \& Preibisch 1994 and Stelzer et al. 2005, 2006, 2009). On account of that similarity, a popular interpretation was that the X-ray emission was in fact due to the presence of a young low-mass stellar companion (rather than to the HAeBe star itself). The hot X-ray component of the single HAeBe star HD 163296, on the other hand, suggested a coronal origin and is the most promising case of an HAeBe hosting a corona (Günther \& Schmitt 2009). To our knowledge, nonthermal radio emission from young intermediate mass stars has only been detected directly, using the VLBI technique, from the B4V S1 star in the Ophiuchus cloud (Andre et al. 1991; Loinard et al. 2008) and from the proto-HAeBe binary star EC 95 in the Serpens cloud (Dzib et al. 2010). The high variability of EC 95 at radio frequencies strongly suggests that it has a coronal origin (see Dzib et al. 2010 for a discussion), while the low variability in $\mathrm{S} 1$ suggests a different origin. However, a corona has never been directly observed from a well established HAeBe star. Future VLBI observations of GBS-VLA J041840.62+281915.3 will establish if the non-thermal radio emission observed with the VLA comes from the HAeBe star itself or from its low-mass

\footnotetext{
$12 \mathrm{HAeBe}$ stars are pre-main sequence intermediate mass stellar objects, i.e.,
} the intermediate mass analogs of T Tauri stars. companion. Even the detection of the companion with the VLBI technique is of great interest because we will be able to estimate the dynamical mass of the HAeBe star.

\subsection{GBS-VLA J045545.85+303304.0 = AB Aur}

The lack of variability and spectral index $(1.27 \pm 0.48)$ for this Herbig Ae star suggests that the emission is produced by a thermal jet. This suggestion is confirmed by the study of Rodríguez et al. (2014b) that combines additional VLA data at other wavelengths with our results.

\section{CONCLUSIONS AND PERSPECTIVES}

We presented a multi-epoch VLA survey at two frequencies of one of the closest and best studied star-forming region, the Taurus-Auriga cloud. It is more sensitive, covers a larger region, and has higher angular resolution than all previous surveys of this region. The multi-epoch, two frequency strategy has enabled us to determine the radio properties of the detected sources, and has provided clues about the nature of their radio emission and the nature of the objects. We detected a total of 610 radio sources, 59 of them related to YSOs and 18 to field stars. We argue that most of the remaining objects are extragalactic sources, but we provide a list of sources whose radio characteristics would be consistent with their being YSOs. Up to half of them may be previously unidentified YSOs.

The radio emission for most of the YSOs (56\%), is consistent with a non-thermal origin (gyrosynchrotron); these sources could be used for future VLBI observations. In line with our studies of other star-forming regions like Ophiuchus, Serpens and Orion (Dzib et al. 2013a; Ortiz-León et al. 2014; Kounkel et al. 2014), we find in Taurus that the radio emission tends to be of a more non-thermal nature for the more evolved YSOs. Finally, by comparing our results with previous X-ray observations we obtained that the sources in Taurus-Auriga follow the so-called Güdel-Benz relation with $\kappa=0.03$, however with a somewhat large dispersion, also consistent with the results in other star-forming regions.

L.L., L.F.R., G.N.O., G.P., and J.L.R. acknowledge the financial support of DGAPA, UNAM, and CONACyT, México. N.J.E. was supported in part by NSF Grant AST-1109116 to the University of Texas at Austin. The National Radio Astronomy Observatory is operated by Associated Universities Inc. under cooperative agreement with the National Science Foundation. CASA is developed by an international consortium of scientists based at the National Radio Astronomical Observatory (NRAO), the European Southern Observatory (ESO), the National Astronomical Observatory of Japan (NAOJ), the CSIRO Australia Telescope National Facility (CSIRO/ATNF), and the Netherlands Institute for Radio Astronomy (ASTRON) under the guidance of NRAO. This research has made use of the SIMBAD database, operated at CDS, Strasbourg, France

\section{REFERENCES}

Adelman-McCarthy, J. K. 2011, yCat, 2306, 0

Andre, P., Phillips, R. B., Lestrade, J.-F., \& Klein, K.-L. 1991, ApJ, 376, 630 Andrews, S. M., \& Williams, J. P. 2005, ApJ, 631, 1134

Anglada, G., Rodriguez, L. F., Canto, J., Estalella, R., \& Torrelles, J. M. 1992, ApJ, 395, 494

Anglada, G., Villuendas, E., Estalella, R., et al. 1998, AJ, 116, 2953

Bally, J., Feigelson, E., \& Reipurth, B. 2003, ApJ, 584, 843

Becker, R. H., \& White, R. L. 1985, ApJ, 297, 649

Benz, A. O., \& Guedel, M. 1994, A\&A, 285, 621 
Bontemps, S. 1996, PhD thesis, Univ. Paris XI

Briceno, C., Calvet, N., Gomez, M., et al. 1993, PASP, 105, 686

Briceño, C., Calvet, N., Kenyon, S., \& Hartmann, L. 1999, AJ, 118, 1354

Briceño, C., Luhman, K. L., Hartmann, L., Stauffer, J. R., \& Kirkpatrick, J. D. 2002, ApJ, 580, 317

Carroll, T. A., Strassmeier, K. G., Rice, J. B., \& Künstler, A. 2012, A\&A, 548, A95

Chini, R., Ward-Thompson, D., Kirk, J. M., et al. 2001, A\&A, 369, 155

Cohen, M., \& Bieging, J. H. 1986, AJ, 92, 1396

Condon, J. J., Cotton, W. D., Greisen, E. W., et al. 1998, AJ, 115, 1693

Connelley, M. S., \& Greene, T. P. 2010, AJ, 140, 1214

Cutri, R. M. 2013, yCat, 2328, 0

Cutri, R. M., Skrutskie, M. F., van Dyk, S., et al. 2003, The IRSA 2MASS All-Sky Point Source Catalog, NASA/IPAC Infrared Science Archive http://irsa.ipac.caltech.edu/applications/Gator/

Dallacasa, D., Stanghellini, C., Centonza, M., \& Fanti, R. 2000, A\&A, 363,887

Damiani, F., Micela, G., Sciortino, S., \& Harnden, F. R., Jr. 1994, ApJ, 436, 807

Deller, A. T., Forbrich, J., \& Loinard, L. 2013, A\&A, 552, A51

Devor, J., Charbonneau, D., O’Donovan, F. T., Mandushev, G., \& Torres, G. 2008, AJ, 135, 850

Dobashi, K., Uehara, H., Kandori, R., et al. 2005, PASJ, 57, 1

Donati, J.-F., Jardine, M. M., Gregory, S. G., et al. 2008, MNRAS, 386, 1234

Doppmann, G. W., Najita, J. R., \& Carr, J. S. 2008, ApJ, 685, 298

Duchêne, G., Bouvier, J., Bontemps, S., André, P., \& Motte, F. 2004, A\&A, 427, 651

Dzib, S., Loinard, L., Mioduszewski, A. J., et al. 2010, ApJ, 718, 610

Dzib, S. A., Loinard, L., Mioduszewski, A. J., et al. 2013a, ApJ, 775, 63

Dzib, S., Loinard, L., Rodríguez, L. F., Mioduszewski, A. J., \& Torres, R. M. 2011, ApJ, 733, 71

Dzib, S. A., Loinard, L., Rodríguez, L. F., \& Galli, P. 2014, ApJ, 788, 162

Dzib, S. A., Rodríguez, L. F., Araudo, A. T., \& Loinard, L. 2013b, RMxAA, 49,345

Elias, J. H. 1978, ApJ, 224, 857

Feigelson, E. D., Carkner, L., \& Wilking, B. A. 1998, ApJL, 494, L215

Feigelson, E. D., \& Montmerle, T. 1999, ARA\&A, 37, 363

Forbrich, J., Massi, M., Ros, E., Brunthaler, A., \& Menten, K. M. 2007, A\&A, 469, 985

Forbrich, J., Posselt, B., Covey, K. R., \& Lada, C. J. 2010, ApJ, 719, 691

Furlan, E., Luhman, K. L., Espaillat, C., et al. 2011, ApJS, 195, 3

Gagné, M., Skinner, S. L., \& Daniel, K. J. 2004, ApJ, 613, 393

Giardino, G., Favata, F., Micela, G., \& Reale, F. 2004, A\&A, 413, 669

Giovanardi, C., Rodríguez, L. F., Lizano, S., \& Cantó, J. 2000, ApJ, 538, 728

Güdel, M., Briggs, K. R., Arzner, K., et al. 2007, A\&A, 468, 353

Guedel, M., \& Benz, A. O. 1993, ApJL, 405, L63

Günther, H. M., \& Schmitt, J. H. M. M. 2009, A\&A, 494, 1041

Gutermuth, R. A., Megeath, S. T., Myers, P. C., et al. 2009, ApJS, 184, 18

Hartigan, P., \& Kenyon, S. J. 2003, ApJ, 583, 334

Hartigan, P., Strom, K. M., \& Strom, S. E. 1994, ApJ, 427, 961

Hartmann, L. 2002, ApJ, 578, 914

Heeschen, D. S. 1984, AJ, 89, 1111

Heeschen, D. S., \& Rickett, B. J. 1987, AJ, 93, 589

Hernández, J., Calvet, N., Briceño, C., Hartmann, L., \& Berlind, P. 2004, AJ, 127,1682
Kenyon, S. J., Gómez, M., \& Whitney, B. A. 2008, in Handbook of Star Forming Regions, ed. B. Reipurth (Volume I: The Northern Sky San Francisco, CA: ASP Monograph Publications), 405

Kenyon, S. J., \& Hartmann, L. 1995, ApJS, 101, 117

Koay, J. Y., Bignall, H. E., Macquart, J.-P., et al. 2011, A\&A, 534, L1

Kounkel, M., Hartmann, L., Loinard, L., et al. 2014, ApJ, 790, 49

Leinert, C., Richichi, A., \& Haas, M. 1997, A\&A, 318, 472

Loinard, L., Mioduszewski, A. J., Rodríguez, L. F., et al. 2005, ApJL, 619, L179

Loinard, L., Rodríguez, L. F., D’Alessio, P., Rodríguez, M. I., \& González, R. F. 2007a, ApJ, 657, 916

Loinard, L., Torres, R. M., Mioduszewski, A. J., \& Rodríguez, L. F. 2008, ApJL, 675, L29

Loinard, L., Torres, R. M., Mioduszewski, A. J., et al. 2007b, ApJ, 671, 546

Lovell, J. E. J., Rickett, B. J., Macquart, J.-P., et al. 2008, ApJ, 689, 108

Manoj, P., Bhatt, H. C., Maheswar, G., \& Muneer, S. 2006, ApJ, 653, 657

Martín, E. L., \& Magazzù, A. 1999, A\&A, 342, 173

Neuhaeuser, R., Sterzik, M. F., Schmitt, J. H. M. M., Wichmann, R., \& Krautter, J. 1995, A\&A, 297, 391

O'Dea, C. P. 1998, PASP, 110, 493

O’Neal, D., Feigelson, E. D., Mathieu, R. D., \& Myers, P. C. 1990, AJ, 100,1610

Orienti, M., \& Dallacasa, D. 2014, MNRAS, 438, 463

Ortiz-León, G., Loinard, L., Mioduszewski, A. J., et al. 2014, ApJ, submitted

Padgett, D., McCabe, C., Rebull, L., et al. 2007, BAAS, 39, 780

Perley, R. A., \& Butler, B. J. 2013, ApJS, 204, 19

Prato, L., Simon, M., Mazeh, T., Zucker, S., \& McLean, I. S. 2002, ApJL, 579, L99

Rebull, L. M., Padgett, D. L., McCabe, C.-E., et al. 2010, ApJS, 186, 259

Reipurth, B., Rodríguez, L. F., Anglada, G., \& Bally, J. 2004, AJ, 127, 1736

Riviere-Marichalar, P., Ménard, F., Thi, W. F., et al. 2012, A\&A, 538, L3

Rodriguez, L. F., Anglada, G., \& Raga, A. 1995, ApJL, 454, L149

Rodríguez, L. F., Carrasco-González, C., Montes, G., \& Tapia, M. 2014a, AJ, 148, 20

Rodríguez, L. F., Dzib, S. A., Loinard, L., et al. 2012a, RMxAA, 48, 243

Rodríguez, L. F., González, R. F., Raga, A. C., et al. 2012b, A\&A, 537, A123

Rodríguez, L. F., \& Reipurth, B. 1998, RMxAA, 34, 13

Rodríguez, L. F., Zapata, L. A., Dzib, S. A., et al. 2014b, ApJL, 793, L21

Skinner, S. L., Brown, A., \& Stewart, R. T. 1993, ApJS, 87, 217

Smith, K. W., Balega, Y. Y., Duschl, W. J., et al. 2005, A\&A, 431, 307

Stelzer, B., Flaccomio, E., Montmerle, T., et al. 2005, ApJS, 160, 557

Stelzer, B., Micela, G., Hamaguchi, K., \& Schmitt, J. H. M. M. 2006, A\&A, 457, 223

Stelzer, B., Robrade, J., Schmitt, J. H. M. M., \& Bouvier, J. 2009, A\&A, 493, 1109

Torres, R. M., Loinard, L., Mioduszewski, A. J., \& Rodríguez, L. F. 2007, ApJ, 671,1813

Torres, R. M., Loinard, L., Mioduszewski, A. J., \& Rodríguez, L. F. 2009, ApJ, 698, 242

Torres, R. M., Loinard, L., Mioduszewski, A. J., et al. 2012, ApJ, 747, 18

Voges, W., Aschenbach, B., Boller, T., et al. 1999, A\&A, 349, 389

Wahhaj, Z., Cieza, L., Koerner, D. W., et al. 2010, ApJ, 724, 835

White, R. J., \& Ghez, A. M. 2001, ApJ, 556, 265

White, R. J., \& Hillenbrand, L. A. 2004, ApJ, 616, 998

Zinnecker, H., \& Preibisch, T. 1994, A\&A, 292, 152 